\title{
The Role of Galactic Winds on Molecular Gas Emission from Galaxy Mergers
}

\section{Citation}

Narayanan, Desika, Thomas J. Cox, Brandon Kelly, Romeel Davé, Lars Hernquist, Tiziana Di Matteo, Philip F. Hopkins, Craig Kulesa, Brant Robertson, and Christopher K. Walker. 2008. "The Role of Galactic Winds on Molecular Gas Emission from Galaxy Mergers." The Astrophysical Journal Supplement Series 176 (2): 331-54. https://doi.org/10.1086/533500.

\section{Permanent link}

http://nrs.harvard.edu/urn-3:HUL.InstRepos:41381686

\section{Terms of Use}

This article was downloaded from Harvard University's DASH repository, and is made available under the terms and conditions applicable to Open Access Policy Articles, as set forth at http:// nrs.harvard.edu/urn-3:HUL.InstRepos:dash.current.terms-of-use\#OAP

\section{Share Your Story}

The Harvard community has made this article openly available. Please share how this access benefits you. Submit a story.

Accessibility 
Submitted to ApJ, September 18th, 2007, ACCePteed JAn 21St, 2008

Preprint typeset using $\mathrm{LT}_{\mathrm{E}} \mathrm{X}$ style emulateapj v. 12/14/05

\title{
THE ROLE OF GALACTIC WINDS ON MOLECULAR GAS EMISSION FROM GALAXY MERGERS
}

\author{
Desika Narayanan ${ }^{1,6}$, Thomas J. CoX ${ }^{2}$, Brandon Kelly ${ }^{1}$, Romeel DaVÉ ${ }^{1}$, Lars Hernquist ${ }^{2}$, Tiziana Di Matteo ${ }^{5}$, Philip \\ F. HOPKINS ${ }^{2}$, Craig KulesA ${ }^{1}$, Brant Robertson ${ }^{3,4}$, Christopher K. WALKeR ${ }^{1}$ \\ Submitted to ApJ, September 18th, 2007, Accepteed Jan 21st, 2008
}

\begin{abstract}
Galactic winds from starbursts and Active Galactic Nuclei (AGN) are thought to play an important role in driving galaxies along the starburst-AGN sequence. Here, we assess the impact of these winds on the $\mathrm{CO}$ emission from galaxy mergers, and, in particular, search for signatures of starburst and AGN-feedback driven winds in the simulated CO morphologies and emission line profiles. We do so by combining a 3D non-LTE molecular line radiative transfer code with smoothed particle hydrodynamics (SPH) simulations of galaxy mergers that include prescriptions for star formation, black hole growth, a multiphase interstellar medium (ISM), and the winds associated with star formation and black hole growth. Our main results are: (1) Galactic winds can drive outflows of masses $\sim 10^{8}-10^{9} \mathrm{M}_{\odot}$ which may be imaged via $\mathrm{CO}$ emission line mapping. (2) AGN feedback-driven winds are able to drive detectable $\mathrm{CO}$ outflows for longer periods of time than starburstdriven winds owing to the greater amount of energy imparted to the ISM by AGN feedback compared to star formation. (3) Galactic winds can control the spatial extent of the $\mathrm{CO}$ emission in post-merger galaxies, and may serve as a physical motivation for the sub-kiloparsec scale $\mathrm{CO}$ emission radii observed in local advanced mergers. (4) Secondary emission peaks at velocities greater than the circular velocity are seen in the $\mathrm{CO}$ emission lines in all models, regardless of the associated wind model. In models with winds, however, these high velocity peaks are seen to preferentially correspond to outflowing gas entrained in winds, which is not the case in the model without winds. The high velocity peaks seen in models without winds are typically confined to velocity offsets (from the systemic) $\lesssim 1.7$ times the circular velocity, whereas the models with AGN feedback-driven winds can drive high velocity peaks to $\sim 2.5$ times the circular velocity.
\end{abstract}

Subject headings: galaxies: active, starburst, ISM - quasars: general - line: profiles - ISM: jets and outflows

\section{INTRODUCTION}

Observed relationships in galaxies between central black hole mass and stellar mass (e.g. Magorrian et al. 1998), velocity dispersion (i.e. the $M_{B} H-\sigma$ relation; e.g. Gebhardt et al. 2000; Ferrarese \& Merritt 2000), or galaxy structural properties (e.g. the black hole fundamental plane: Hopkins et al. 2007a,b) indicate a co-eval nature in supermassive black hole growth and star formation in galaxies. These results have prompted a number of investigations in recent years to quantify this apparent self-regulation in star formation and black hole growth in galaxies, and their relationship to the formation and evolutionary history of the host system (e.g. Kauffmann \& Haehnelt, 2000; Hopkins et al. 2006a,b; 2007c,d).

Over the last two decades, observations of local galaxies have painted a compelling picture in which galaxy mergers provide the link between massive starbursts and central black hole growth and activity. ULIRGs (Ultraluminous Infrared Galaxies), for example, are a class of starburst galaxies with elevated infrared luminosities $\left(\mathrm{L}_{\mathrm{IR}} \geq 10^{12} \mathrm{~L}_{\odot}\right)$ which typically show signs of interactions (e.g. Downes \& Solomon, 1998; Sanders et al. 1988a; Scoville et al. 2000). While the intense infrared luminosity in these sources certainly owes in

\footnotetext{
${ }^{1}$ Steward Observatory, University of Arizona, 933 N Cherry Ave, Tucson, $\mathrm{Az}, 85721$, USA

${ }^{2}$ Harvard-Smithsonian Center for Astrophysics, 60 Garden Street, Cambridge, MA 02138, USA

${ }^{3}$ Kavli Institute for Cosmological Physics and Department of Astronomy and Astrophysics, University of Chicago, 933 East 56th St., Chicago, Il, 60637

${ }^{4}$ Spitzer Fellow

${ }^{5}$ Carnegie Mellon University, Department of Physics, 5000 Forbes Ave., Pittsburgh, PA 15213

${ }^{6}$ dnarayanan@as.arizona.edu
}

large part to the merger induced starbursts (e.g. Sanders et al. 1988a,b; Sanders \& Mirabel, 1996), in some cases a contribution from a buried active galactic nucleus (AGN) may be nonnegligible. For example, many ULIRGs show spectral energy distributions (SEDs), infrared color ratios (e.g. F[25/60 $\mu \mathrm{m}]$ ), polycyclic aromatic hydrocarbon emission (PAH) deficits, and emission line fluxes (e.g. [Ne V] at $14.3 \mu \mathrm{m}$ ) consistent with central AGN activity (e.g. Armus et al. 2004, 2006; Farrah et al. 2003; de Grijp et al. 1985). Indeed, 35-50\% of ULIRGs with luminosity above $\mathrm{L}_{\mathrm{IR}}$ of $10^{12.3} \mathrm{~L}_{\odot}$ show optical and NIR spectra consistent with AGN activity (Kim, Veilleux \& Sanders, 2002; Tran et al. 2001; Veilleux, Kim \& Sanders, 1998). These results suggest that these merging systems are simultaneously undergoing a massive star formation and central black hole growth phase.

Observed similarities such as these between starburst galaxies, ULIRGs and quasars prompted Soifer et al. (1987) and Sanders et al. (1988a,b) to propose an empirically derived evolutionary sequence which connects galactic starbursts to quasars through galaxy mergers. In this picture, the fueling of the central black hole and AGN growth phase is realized during the major merger when gaseous inflows trigger nuclear starbursts as well as central black hole growth. The dusty galaxy transitions from a cold, starburst dominated ULIRG, to a warm, AGN-dominated ULIRG (where 'cold' and 'warm' refer to $\mathrm{F}[25 / 60 \mu \mathrm{m}]$ ratios), and, as supernovae and stellar winds clear the obscuring gas and dust, to an optical quasar. In this sense, galaxy mergers provide a unique laboratory for studying the possible co-evolution of starbursts, black hole growth and activity, and spheroid formation.

Recent models have provided a theoretical foundation and further evidence for a merger-driven starburst-AGN connection in galaxies. Specifically, simulations by Springel, Di 
Matteo \& Hernquist (2005a) have shown that galaxy mergers can fuel large-scale gaseous inflows (e.g. Barnes \& Hernquist, 1991, 1996) which trigger nuclear starbursts (Mihos \& Hernquist, 1996; Springel et al. 2005a) as well as promote central black hole growth (Di Matteo, Springel \& Hernquist, 2005). Subsequent winds associated with the growth of central black holes can lift the veil of obscuring gas and dust, and along several sightlines produce a quasar with comparable lifetimes, luminosity functions, and observed $B$-band and $\mathrm{X}$-ray properties to those observed (Cox et al., 2006b; Hopkins et al. 2005a-d; 2006a-d). The merger remnants quickly redden owing to gas depletion and the impact of feedback from star formation and black hole growth (e.g. Springel et al. 2005b) and resemble elliptical galaxies in their kinematic and structural properties. Indeed, the population of stars formed during the starbursts accounts for the central "excess light" seen in ongoing mergers (e.g. Rothberg \& Joseph 2004, 2006) and provides a detailed explanation for the luminosity profiles of old ellipticals (e.g. Kormendy et al. 2007; see Mihos \& Hernquist 1994a; Hopkins et al. 2007e,f,g).

A consensus picture has thus been borne out from these observations and simulations over the last two decades in which so called 'feedback' processes associated with winds from star formation and black holes act to self-regulate the growth of both the stellar and black hole masses in galaxies (e.g. Fabian, 1999; Silk \& Rees, 1998). Indeed, the effects of starburst and AGN feedback-driven winds have been observed in local galaxies (e.g. Heckman et al. 2000; Martin, 2005; Rupke, Veilleux \& Sanders, 2005a-c; Rupke \& Veilleux, 2005; Tremonti, Moustakas \& Diamond-Stanic, 2007), as well as those at high-z (e.g. Narayanan et al. 2004; Pettini et al. 2002; Shapley et al. 2003) by way of absorption line outflows. However, the direct effect of feedback processes (especially from the highly efficient central AGN) on the emission properties of galaxies is not yet well characterized (see Veilleux, Cecil \& Bland-Hawthorn, 2005 for an extensive review and associated references). In this sense, it is important to relate theories of galaxy formation and evolution which incorporate physically motivated models of feedback processes to observational signatures of winds across the electromagnetic spectrum.

In particular, observations of molecular gas in galaxies have proven valuable in characterizing the physics related to nuclear star formation and central AGN fueling in galaxy mergers as the molecular gas serves as fuel both for star formation as well as the central black hole(s). For example, interferometric observations of molecular gas in ULIRGs show that most typically harbor $\sim 10^{10} \mathrm{M}_{\odot}$ of molecular gas within the central $1.5 \mathrm{kpc}$ (Scoville et al. 1986; Bryant \& Scoville, 1999). Moreover, high resolution maps of dense molecular gas at submillimeter wavelengths in ULIRGs have revealed kinematic structures of double nuclei in mergers (Scoville, Yun \& Bryant, 1997), bar-driven inflows (Sakamoto et al. 2004), and the density structure of gas fueling the central AGN (Iono et al. 2004).

With the increased spatial resolution and sensitivity afforded by the latest generation of (sub)mm-wave interferometers (e.g. the SMA, CARMA, PdBI), a number of recent observations have been able to pioneer investigations as to the effects of galactic winds on molecular gas emission in galaxies (e.g. Iono et al. 2007; Sakamoto, Ho \& Peck, 2006; Walter, Weiß \& Scoville, 2002). Detections such as these are expected to become more routine in upcoming years as the ALMA interferometer becomes available. An important complement to these current and forthcoming observations of molecular line emission from starburst galaxies and AGN are physical models which directly relate $\mathrm{CO}$ emission properties to galactic scale winds.

In this context, it is our aim to investigate the role that galactic winds can play on $\mathrm{CO}$ emission properties from starburst galaxies and AGN via numerical simulations. In particular, we focus on specific signatures of winds imprinted on $\mathrm{CO}$ morphologies and emission line profiles. In this work, we present self-consistent radiative transfer calculations for the emission properties of $\mathrm{CO}$ molecular gas in gas-rich galaxy mergers which account for the winds associated with both star formation and black hole growth.

In $\S 2.1$, we describe the hydrodynamic simulations, and radiative transfer methodology. In $\S 3$ we provide an example of our methods by applying our radiative transfer calculations to a model of a star-forming disk galaxy. In $\$$ 4 we discuss the effect of winds on observed CO morphologies. We explore the response of emission line profiles to winds in $\S 5$ present a broader discussion of these results with respect to observations in $\S 6$, and summarize in $\S 7$. Throughout this paper, we assume a $\Lambda$ CDM cosmology with $h=0.7, \Omega_{\Lambda}=0.7$, $\Omega_{\mathrm{M}}=0.3$.

\section{METHODOLOGY}

Simulating molecular line emission on galaxy-wide scales has a relatively short history owing to the computational costs associated with the hydrodynamics and radiative transfer. Early works by Silk \& Spaans (1997) and Combes, Maoli $\&$ Omont (1999) focused on simulating molecular emission from high- $z$ objects, using idealized galaxy systems. Wada \& Tomisaka (2005) pioneered incorporating three-dimensional non local thermodynamic equilibrium (non-LTE) radiative transfer calculations into hydrodynamic simulations of AGN, focusing specifically on predicted emission from the circumnuclear molecular torus. More recent works have folded non-LTE radiative transfer codes into self-consistent hydrodynamic simulations of galaxy mergers. These works have been performed in mergers scaled for low redshift (Narayanan et al. 2006a), as well as those appropriate for higher- $z$ systems (Greve \& Sommer-Larsen, 2006; Narayanan et al. 2007). Here, we employ a similar methodology to that of Narayanan et al. (2006a, 2007). In this section, we describe our methods for incorporating 3D non-LTE molecular line radiative transfer calculations into hydrodynamic simulations of galaxy mergers which account for a multi-phase ISM, star formation, growing black holes, and the effects of winds associated with star formation and central AGN.

\subsection{Numerical Methods}

\subsubsection{Hydrodynamics}

The hydrodynamic simulations used for this study employed a modified version of the publicly available $N$ body/SPH code, GADGET-2 (Springel, 2005), adopting the fully conservative formulation of SPH developed by Springel $\&$ Hernquist (2002). The methods used to construct the progenitor galaxies, as well as a detailed description of the algorithms used to simulate the physics of feedback from star formation and accreting black holes is detailed in Springel, Di Matteo \& Hernquist (2005a), and we direct the reader toward this work for further information. Here, we briefly summarize these methods, and describe the specifics of the modeling most pertinent to this work. 
The interstellar medium (ISM) is modeled as a multi-phase medium in which pressure feedback from supernovae heating is treated through an effective equation of state (EOS) (Springel, Di Matteo \& Hernquist, 2005a). The disk galaxies used as progenitors in these simulations employed a softened EOS with softening parameter $q_{\mathrm{EOS}}=0.25$ (see Figure 4 of Springel et al. 2005a). The ISM is modeled to include cold, dense gas surrounded by a hot ISM, and is realized numerically through "hybrid" SPH particles (Springel \& Hernquist, 2003). In this formulation, cold ISM is allowed to grow through radiative cooling of the hot ISM, and conversely feedback associated with star formation can evaporate cold clouds into diffuse, hot gas. Star formation follows the prescription of Springel \& Hernquist (2003) and is constrained to fit the Schmidt/Kennicutt observed star formation laws (Kennicutt, 1998a,b; Schmidt, 1959).

In order to explore the effects of galactic winds on the molecular ISM, we include a formulation for both accreting black holes, and winds associated with massive starbursts. In our SPH formalism, the black holes are included as sink particles which accrete gas from the surrounding ISM. The accretion is treated with a Bondi-Lyttleton-Hoyle parametrization (Bondi \& Hoyle, 1944, Hoyle \& Lyttleton, 1939) with a fixed maximum rate corresponding to the Eddington limit. The black hole radiates such that its bolometric luminosity is given by $L=\epsilon \dot{M} c^{2}$ with accretion efficiency $\epsilon=0.1$. We further assume that $5 \%$ of this energy couples to the surrounding ISM such that $0.5 \%$ of the accreted mass energy in our simulations is reinjected isotropically into the ISM as thermal energy (a parameter choice which allows the merged galaxy to reproduce the local $M_{B} H-\sigma$ normalization [Di Matteo, Springel \& Hernquist, 2005]). Hence, the black hole energy deposition rate into the ISM may be expressed as

$$
\dot{E}_{\mathrm{BH}}=\epsilon_{\text {effective }} \dot{M}_{\mathrm{BH}} c^{2}
$$

where $\epsilon_{\text {effective }}=0.005$ (Cox et al. 2007; Springel, Di Matteo \& Hernquist., 2005a).

Starburst winds are treated utilizing the constant wind models of Springel \& Hernquist (2003). In this formulation, the mass loss rate (denoted by $\dot{M}_{w}$ ) is assumed to be proportional to the star formation rate (denoted by $\dot{M}_{\star}$ ), such that:

$$
\dot{M}_{w}=\eta \dot{M}_{\star}
$$

where $\eta$ quantifies the wind mass loading factor. The wind is assumed to have a speed constrained by a fraction of the supernova energy $(\chi)$ :

$$
\frac{1}{2} \dot{M}_{w} v_{w}^{2}=\chi \epsilon_{\mathrm{SN}} \dot{M}_{\star}
$$

In a recent study of galactic winds in galaxy mergers, Cox et al. (2007) found that winds with high mass loading factors $(\eta \geq 1)$ may be unphysical in that they tend to prevent any starburst after the major merger. We thus choose a moderate mass loading factor of $\eta=0.5$, and constant wind velocity $v_{w}=837 \mathrm{~km} \mathrm{~s}^{-1}$ for this study. These numbers are consistent with the favored momentum-driven wind scalings for the galaxy masses we simulate found by Oppenheimer \& Davé (2006). For more details concerning constraints on wind velocities and efficiencies in galactic scale simulations, we refer the reader to Cox et al. (2007).

The energy input rate into the ISM from star formation may be expressed as

$$
\dot{E_{\mathrm{sb}}}=\epsilon_{\mathrm{SN}} \dot{M_{\star}}
$$

where $\epsilon_{\mathrm{SN}}$ is the energy imparted by supernovae, per solar mass, taken here to be $1.4 \times 10^{49}$ ergs $\mathrm{M}_{\odot}{ }^{-1}$ (Springel \& Hernquist 2003).

The progenitor galaxies in our models contain a dark matter halo initialized with a Hernquist (1990) profile, concentration index $c=9$, spin parameter $\lambda=0.033$, circular velocity $V_{200}=160 \mathrm{~km} \mathrm{~s}^{-1}$, and are bulge-less. The galaxy's exponential disk is rotationally supported and comprises $4.1 \%$ of the total mass. We utilize 120,000 dark matter particles, and 80,000 total disk particles, $40 \%$ of which are gaseous in nature, the remainder serving as collisionless star particles. The total masses of the progenitor galaxies were $1.4 \times 10^{12} \mathrm{M}_{\odot}$, and the final merger produced a central black hole with mass $\sim 5 \times 10^{7} \mathrm{M}_{\odot}$. The softening lengths were $100 \mathrm{pc}$ for baryons, and $200 \mathrm{pc}$ for dark matter.

We have conducted five binary merger simulations. Each of the progenitor galaxies in our simulations was constructed with the aforementioned physical parameters, and the varied parameters include initial orbit and types of wind included (e.g. starburst and/or AGN). Identical progenitor galaxies were used in each simulation. The initial conditions for the merger simulations are summarized in Table 1, and we will henceforth refer to the simulations by their model name listed in Column 1 of Table 1 . In order to simplify analysis throughout this paper we will largely analyze the properties of the four simulations which share the same merger orientation and utilize the coplanar simulations (model co-BH) for only specific comparisons.

\subsubsection{Radiative Transfer}

Submillimeter and millimeter wave radiation from molecules is dependent on the distribution of level populations which depend both on collisions with other molecules and atoms, as well as the incident radiation field. The excitation of molecular gas is highly sensitive to both variations in the temperature and density distribution, as well as the incident radiation field. There are usually large differences in the collisional densities necessary to excite different energy levels. For example, in the case of $\mathrm{CO}$, the critical density to collisionally populate the $\mathrm{J}=1$ state is $n_{\text {crit }} \sim 10^{2}-10^{3} \mathrm{~cm}^{-3}$ while the $\mathrm{J}=3$ state typically requires $n_{\text {crit }} \sim 10^{4} \mathrm{~cm}^{-3}$. The latter density is characteristic of dense cores in GMCs, whereas the former is typical of diffuse GMC atmospheres.

The non-LTE radiative transfer calculations for this work were calculated during post-processing of the hydrodynamic simulations. The spatial range considered for the radiative transfer was $12 \mathrm{kpc}$. The simulation outputs were smoothed to a resolution of $\sim 250 \mathrm{pc}$ in order to defray computational costs associated with the radiative transfer simulations. In order to model the strongly density-dependent excitation rates in our simulations, we have expanded the Bernes (1979) nonlocal thermodynamic equilibrium (LTE) Monte Carlo radiative transfer algorithm to include a mass spectrum of GMCs in a subgrid manner.

The molecular gas fraction in galaxies is determined by the metallicity, dust content, interstellar radiation field, density and temperature (Hollenbach, Werner \& Salpeter, 1971; Pelupessy, Papadopoulos \& van der Werf, 2006). However, owing to limited spatial resolution, this calculation cannot be done explicitly as the location of individual stars and clouds are not know. We therefore assume that half of the cold neutral gas mass in each grid cell is in atomic form and half in molecular, as motivated by local volume surveys of star forming galaxies (e.g. Keres, Yun \& Young 2003) though note that an esti- 
TABLE 1

Progenitor GALAXiES

\begin{tabular}{ccccccccccc}
\hline \hline Run & Other Names Used & $\theta_{1}$ & $\phi_{1}$ & $\theta_{2}$ & $\phi_{2}$ & $\begin{array}{c}\mathrm{V}_{\text {vir }} \\
\left(\mathrm{km} \mathrm{s}^{-1}\right)\end{array}$ & Gas Frac & BH & SNe \\
\hline BH & $\mathrm{e}$ & 30 & 60 & -30 & 45 & 160 & 0.4 & yes & no \\
no-winds & e-no & 30 & 60 & -30 & 45 & 160 & 0.4 & no & no \\
sb & - & 30 & 60 & -30 & 45 & 160 & 0.4 & no & yes \\
sBH & - & 30 & 60 & -30 & 45 & 160 & 0.4 & yes & yes \\
co-BH & $\mathrm{h}$ & 0 & 0 & 0 & 0 & 160 & 0.4 & yes & no \\
\hline
\end{tabular}

${ }^{a}$ Column 1 is the name of the model used in this work. Column 2 lists alternative names for these models used by Chakrabarti et al. (2007a,b), Cox et al. (2006a,b; 2007), Hopkins et al. (2005a-d; 2006a-d; 2007h), and Robertson et al. (2006a-c), for comparison. Columns $3 \& 4$ are initial orientations for disk 1, Columns 5 $\& 6$ are for disk 2. Column 7 gives the virial velocity of the progenitors and Column 8 gives their initial gas fractions. Columns 9 and 10 describe the types of winds (optionally) included in each model.

mate of the molecular fraction can be obtained via sub-grid prescriptions (e.g. Pelupessy et al. 2006; Greve \& SommerLarsen 2006; Robertson \& Kravtsov 2007).

In our formulation, the cold molecular gas is assumed to be exist in a mass spectrum of GMCs in each grid cell

$$
\frac{d N}{d M} \propto M^{-\beta}
$$

where we take $\beta=1.8$ (Blitz et al. 2006). The GMCs are modeled as power-law spheres where the density $(n)$ is given by:

$$
n=n_{0}\left[\frac{r_{0}}{r}\right]^{\alpha}
$$

The radius of the cloud is determined by the Galactic GMC mass-radius relation (Rosolowsky 2005, 2007; Solomon et al. 1987), such that the sum of the cloud density distributions in a grid cell takes the form:

$$
n=\frac{1}{r_{m}^{\alpha}}\left[\sum_{m} r_{0, m}^{\alpha} n_{0, m}\right]
$$

which is used to set the initial level populations, as well as the densities involved in the collisional rate calculations. For this work we utilize a cloud power-law index of $\alpha=2$ (Walker, Adams \& Lada 1990). Observational evidence suggests that a range of power law indices in GMCs from $\alpha=1-2$ may be appropriate (Andre, Ward-Thompson \& Motte 1996; Fuller \& Myers 1992; Ward-Thompson et al. 1994), although our results are not very sensitive to the choice of power law index within this range.

Formally, we build an emergent spectrum by integrating the equation of radiative transfer along the line of sight:

$$
I_{\nu}=\sum_{z_{0}}^{z} S_{\nu}(z)\left[1-e^{-\tau_{\nu}(z)}\right] e^{-\tau_{\nu}(\mathrm{tot})}
$$

where $I_{\nu}$ is the frequency dependent intensity, $S_{\nu}$ is the source function, $\tau$ is the optical depth, and $z$ is the position along the line of sight.

If the level populations are in LTE, then the source function, $S_{\nu}$ can be simply replaced by the Planck function. However, when considering the propagation of lines through a medium with insufficient density for collisions to thermalize the level populations (e.g., $n \ll n_{\text {crit }}$ ), the effects of radiative excitation and de-excitation must be considered. In this case, the source functions must be calculated explicitly.

The source function from each cloud, $m$, for a given transition from upper level to lower level $u \rightarrow l$ is given by

$$
S_{\nu, m}=\frac{n_{u, m} A_{u l}}{\left(n_{l, m} B_{l u}-n_{u, m} B_{u l}\right)}
$$

where the level populations are assumed to be in statistical equilibrium, and determined through the rate equations:

$$
\begin{gathered}
n_{l, m}\left[\sum_{k<l} A_{l k}+\sum_{k \neq l}\left(B_{l k} J_{\nu}+C_{l k}\right)\right]= \\
\sum_{k>l} n_{k, m} A_{k l}+\sum_{k \neq l} n_{k, m}\left(B_{k l} J_{\nu}+C_{k l}\right) .
\end{gathered}
$$

$A, B_{k l}$ and $B_{l k}$ are the Einstein rates for spontaneous emission, stimulated emission, and absorption, respectively, $C$ are the collisional rate coefficients, the indices $l$ and $k$ represent different energy levels, and $J_{\nu}$ is the mean intensity through a given grid cell:

$$
J_{\nu}=\frac{1}{4 \pi} \int I_{\nu} d \Omega
$$

As is evident by the previous three equations, the problem is circular: the observed intensity depends on the source function which is determined by the level populations. When in a non-LTE regime ( $n \ll n_{\text {crit }}$ ), collisions alone do not determine the level populations, but rather the mean intensity field (e.g. radiation from gas in other grid cells) plays a role as well. The solution is achieved by means of iteration.

The initial level populations in a grid cell are estimated based on the global density distribution for all clouds in a grid cell (Equation 7). We emit model photons which represent many real photons from the mass spectrum of GMCs in each grid cell. The model photons are given a weight $W$ proportional to the total number of molecules in the upper state of a given transition in a cell, and the Einstein $A$ rate coefficient for the transition. The photons are emitted isotropically in 3 dimensions and have line frequency randomly drawn from the line profile function:

$$
\phi(\nu)=\frac{1}{\sigma \sqrt{\pi}} \exp \left\{-\left(\nu-\nu_{0}-\mathbf{v} \cdot \hat{\mathbf{n}} \frac{\nu_{\mathbf{u l}}}{\mathbf{c}}\right)^{2} / \sigma^{2}\right\}
$$

where the effects of the local kinetic temperature and microturbulent velocity field are accounted for via

$$
\sigma=\frac{\nu_{0}}{c}\left[\frac{2 k T}{m}+V_{\text {turb }}\right]^{\frac{1}{2}}
$$

The photon then takes a step over distance $s$ through the next grid cell. After taking a step, the weight is attenuated by a factor $e^{-\tau}$ where the opacities are given by

$$
\begin{array}{r}
\alpha_{\nu}(\text { dust })=\kappa_{\nu} \rho_{\text {dust }} \\
\alpha_{\nu}^{u l}(\text { gas })=\frac{\mathrm{h} \nu_{\mathrm{ul}}}{4 \pi} \phi(\nu)\left(\mathrm{n}_{1} \mathrm{~B}_{\mathrm{lu}}-\mathrm{n}_{\mathrm{u}} \mathrm{B}_{\mathrm{ul}}\right)
\end{array}
$$




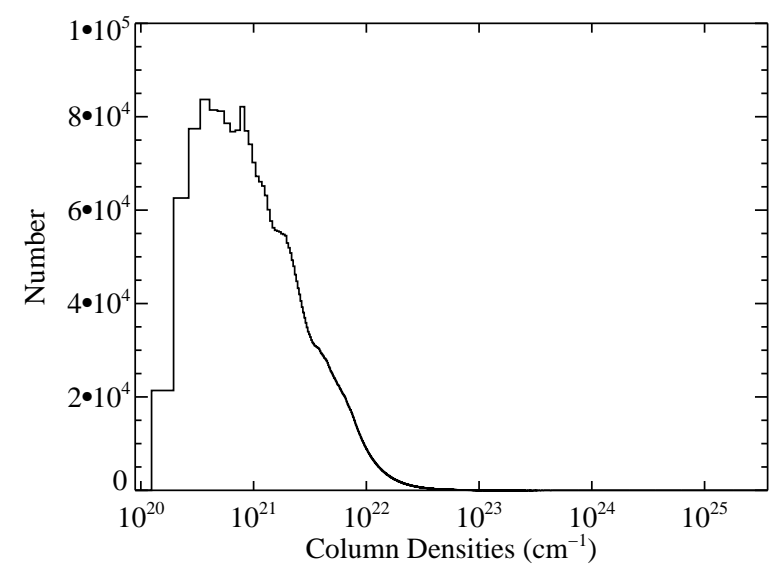

FIG. 1.- Representative distribution of column densities for a grid cell of mass $\sim 3 \times 10^{6} \mathrm{M}_{\odot}$. Distribution of columns is derived by randomly placing clouds drawn from a mass spectrum until the distribution converges. Photons entering a grid cell see a column randomly drawn from a distribution such as this in order to simulate the actual column seen by a photon in a statistical mapner.

$$
\tau_{\nu, m}=\left(\alpha_{\nu, m}(\text { dust })+\alpha_{\nu, \mathrm{m}}(\text { gas })\right) \times \mathrm{s}
$$

The column seen by a photon in a grid cell is calculated via a Monte Carlo approach. Specifically, we calculate the distribution of potential column densities a photon could see through a given grid cell by simulating a sample of the subgrid cells individually on higher resolution $\left(512^{3}\right)$ grids. In these higher resolution grids, we place GMCs at random locations with masses drawn from the power-law mass spectrum, and radii given by the Galactic mass-radius relation. We do this numerous times until the distribution of potential columns a photon entering at random angle sees converges. The distribution of columns is found to scale in a self-similar manner with grid cell (molecular) mass. We then explicitly draw from this distribution to determine the column seen by a model photon as it leaves and enters given grid cells. To illustrate this, we plot an example of the distribution of column densities in Figure 1. The photon continues to propagate through grid cells in this manner until the weight is negligible, or the photon has left the grid.

Once a sufficiently large number of model photons have been emitted to simulate the mean radiation field, $J_{\nu}$, the level populations can be updated via the rate equations. In the rate equations (also commonly called the equations of statistical equilibrium), the terms involving the mean intensity represent the radiatively induced excitations (absorption) and stimulated emission in a given cell of clouds $m^{\prime}$. We may rewrite these equations by defining the number of excitations of a molecule in lower state $l$ by

$$
S_{l u, m^{\prime}}=\frac{h \nu_{u l}}{4 \pi} \phi(\nu) B_{l u} \frac{s W_{0}}{V_{m^{\prime}} \tau}\left(1-e^{-\tau(\mathrm{tot})}\right)
$$

where $V$ is the volume of the cell and $W_{0}$ is the original weight of the photon.

Following Bernes (1979), we then rewrite the equations of statistical equilibrium as

$$
\begin{aligned}
& n_{l}\left[\sum_{k<l} A_{l k}+\sum_{k \neq l}\left(S_{l k, m^{\prime}}+C_{l k}\right)\right]= \\
& \sum_{k>l} n_{k} A_{k l}+\sum_{k \neq l} n_{k}\left[\frac{g_{l}}{g_{k}} S_{l k, m^{\prime}}+C_{k l}\right]
\end{aligned}
$$

where $g$ are the statistical weights of the level.

After a single generation of photons has been emitted, the $S_{l u, m^{\prime}}$ is calculated for each grid cell, $m^{\prime}$. We can then use the analog of the previous equation to calculate the updated level populations for the $N$ individual radial cells that make up the subgrid spectrum of GMCs. The relative contribution of each of the $N$ cells to the total number of excitations $\left(S_{l u, m^{\prime}}\right)$ in a given grid cell is determined via the column across that region.

New level populations are calculated for each of the $N$ cells in the spectrum of GMCs via matrix inversion and then summed to give the total populations for their parent grid cell. New weights $W$ are given to a new generation of emergent photons, and the process is iterated upon until the level populations across the grid are converged. We typically emitted $12 \times 10^{6}$ model photons per iteration and the boundary conditions for the radiative transfer included the $2.73 \mathrm{~K}$ microwave background.

The radiative transfer and excitation depend sensitively on the accuracy of the rate coefficients. We have obtained our coefficients from the Leiden Atomic and Molecular Database (Schoier et al. 2005). We have tested our radiative transfer codes per publicly available benchmarks published in van Zadelhoff et al. (2002). These tests comprise "inside-out" collapsing spherical molecular clouds and the details of the performance of our codes under these standard tests can be found in Figure 1 of Narayanan et al. (2006b).

Owing to its relatively high abundance $\left(\mathrm{CO} / \mathrm{H}_{2}=1.5 \times 10^{-4}\right.$ in the Galaxy; Lee, Bettens \& Herbst, 1996) compared to other common molecules (e.g. $\mathrm{HCN}, \mathrm{CS}$ and $\mathrm{HCO}^{+}$), ${ }^{12} \mathrm{CO}$ (hereafter, $\mathrm{CO}$ ) is the most commonly observed molecular tracer of $\mathrm{H}_{2}$. We therefore focus our modeling efforts for this work on the predicted $\mathrm{CO}$ emission properties of galaxy mergers in the context of galactic winds, and defer investigations of emission from other molecules to future work.

\subsubsection{Model Assumptions}

This methodology has the distinct advantages of being able to simulate the effects of dense cores as well as diffuse atmospheres of clouds. Because we divide the GMCs into a series of sub-grid cells, we are not constrained to using a single density in our rate equations, and thus the collisional excitation characteristic of dense cores can be well-represented while the influence of radiation on more diffuse regions can likewise be accounted for. Moreover, we are able to include these features in our calculations without expanding the grid to computationally prohibitive sizes.

Our subgrid formulation is dependent on a series of assumptions as well. First, owing to limited spatial resolution, we are forced to assume a constant $\mathrm{H}_{2}$ gas fraction constrained by observational surveys. This molecular gas in a given grid cell is assumed to be all in GMCs. The GMCs are realized as power-law spheres in our model whereas clouds are understood observationally to be fractal in nature (e.g. Elmegreen \& Falgarone, 1996). The clouds within each grid cell are all at the same temperature, and individually isothermal (though temperatures are allowed to vary from grid cell to grid cell, and thus temperature gradients exist across the galaxy).

The temperatures in the cold phase of the ISM in the hydrodynamic models are fixed at $1000 \mathrm{~K}$ (Springel \& Hernquist, 2003). This choice is arbitrary and has no effect on the hydrodynamic simulations. Typically, temperatures in molecular clouds range from $10-100 \mathrm{~K}$; temperatures of $1000 \mathrm{~K}$ would systematically put too much weight on the collisional coeffi- 
cients in the rate equations, resulting in overly excited level populations. While it is indeed tractable to run galaxy-wide hydrodynamic models which allow the temperatures to cool to lower values than those set here (e.g. Robertson \& Kravtsov 2007), it is computationally infeasible for the large numbers of simulations presented in this work (Table 1). Thus, a subgrid model for refining the temperature structure of the ISM is required. We construct a relatively simple model for the ISM temperature structure by considering the dominant heating mechanism for the gas as heating from $\mathrm{O}$ and $\mathrm{B}$ stars (assuming a Salpeter IMF). We sample individual grid cells on a finer $\left(512^{3}\right)$ resolution and place $\mathrm{O}$ and $\mathrm{B}$ stars formed in the hydrodynamic simulations in the power-law sphere clouds randomly throughout the higher resolution grid cells. These higher resolution cells are utilized to calculate the mean column of gas and dust (which are assumed to have the same spatial distribution in the clouds) seen by the stellar UV flux, and the mean blackbody heating rate is derived for each grid cell. When no $\mathrm{O}$ stars are present, lower mass stars dominate the heating of the gas. Owing to the large grid cell size $(\sim 250 \mathrm{pc})$, and the high densities of the molecular cores in the subgrid clouds $\left(\gtrsim 10^{5} \mathrm{~cm}^{-3}\right)$, blister regions from the few formed $\mathrm{O}$ stars have a negligible impact on the total molecular gas content (Hollenbach \& Tielens 1999). The neutral gas is further allowed to cool via metal line cooling which is calculated using a mean escape probability radiative transfer code (ESCAPE; Kulesa 2002; Kulesa et al. 2005). The dominant ISM coolants considered are CII, NII and atomic Oxygen and Carbon with abundances set at Galactic values (Lee, Bettens \& Herbst 1996). Heating and excitation from a central AGN (when included) is not considered, and is deferred to a future study.

Utilizing this methodology, we find typical isothermal cloud temperatures ranging from $10 \mathrm{~K}-120 \mathrm{~K}$ (nominally $10-30$ $\mathrm{K}$ for simulations scaled for the local Universe such as those presented in this work, though temperatures can approach $\sim 120 \mathrm{~K}$ for models of higher redshift galaxies; Narayanan et al. 2007a). It is additionally worth noting as well that variations in the temperature structure of the ISM within this range are ancillary with respect to determining the excitation patterns of the molecular ISM. As an example, doubling the temperature from $10 \mathrm{~K}$ to $20 \mathrm{~K}$ only changes the collisional rates of excitation of e.g. $\mathrm{CO} J=2$ to $\mathrm{CO} J=3$ by $\sim 10 \%$ (utilizing values taken from the Leiden Atomic and Molecular Database; Schoier et al. 2005). The dominant effect is instead the density structure of the GMCs. Tests have shown that the excitation conditions of these models are robust while the assumed cloud power-law index and/or GMC mass spectrum index remain within observational constraints (Blitz et al. 2006; Rosolowsky 2007; see also Narayanan et al. 2007b).

\subsubsection{Resolution Tests}

In order to test the convergence properties of the presented results, we have taken a snapshot of merger BH (black hole winds only) at the peak of the quasar phase, and examined the simulated $\mathrm{CO}$ properties at a variety of spatial resolutions. In Figure 2, we plot the $\mathrm{CO}(\mathrm{J}=1-0)$ images of the sample galaxy at three resolutions, and the normalized CO $(\mathrm{J}=1-0)$ spectra at the same resolutions. The detailed structure of the galaxy's morphology begins to become evident at resolutions of $\sim 240 \mathrm{pc}$, though of course shows smaller-scale features at finer resolution. Similarly, at 240 pc resolution the unresolved spectra begin to show detailed kinematic signatures in the line profile that are smoothed over in the lower spatial res- olution simulations. Thus, for both image analysis and spectral line profile analysis we utilize simulations with $\sim 240 \mathrm{pc}$ resolution in order to glean the maximum information while minimizing the extensive computational costs associated with galaxy-wide non-LTE radiative transfer.

Knowledge of the excitation properties of $\mathrm{CO}$ requires a slightly higher spatial resolution. While the work presented here does not nominally investigate the excitation properties of the CO molecular gas to great detail, other investigations utilizing models similar to these (e.g. Narayanan et al. 2007b) examine the excitation properties in greater detail. We therefore briefly review the convergence of the $\mathrm{CO}$ excitation. The excitation properties require a higher spatial resolution for convergence, and we therefore include a higher resolution simulation $(120 \mathrm{pc})$ for comparison. In Figure 3, we plot the ratio of the intensity of the highest resolution run $(120 \mathrm{pc})$ versus lower resolution simulations for a series of $\mathrm{CO}$ transitions. The results for higher lying transitions $(\mathrm{CO} \gtrsim 4)$ are only converged for higher spatial resolution $(\sim 160 \mathrm{pc})$ than is typically required for the morphology or spectral line profile studies. Therefore higher resolution models must be accounted for in studies concentrating on the molecular excitation (e.g. Narayanan et al. 2007b). For the few excitation condition investigations in this work, we employ targeted higher resolution simulations.

\subsection{Overview of A Major Merger}

In the binary merger simulations presented here, after the initial passage of the merging galaxies, gas is driven into the nuclear regions owing to tidal torquing (Barnes \& Hernquist, 1991, 1996). This begins a major black hole growth phase. While the galaxies approach toward and undergo final coalescence $^{7}$ (hereafter defined as the in-spiral stage) they go through a massive starburst and LIRG/ULIRG phase while the central black hole grows as an enshrouded AGN following the methodology developed by Springel et al. (2005a). In the models which include AGN feedback-driven winds, after nuclear coalescence, the thermal energy associated with the black hole accretion drives a powerful wind into the surrounding ISM and strongly reduces the star formation rate. During much of this time, along several sightlines, the object may be viewed as an optical quasar (Hopkins et al. 2005ad; 2006a-d; 2007h). The galaxy then proceeds to evolve passively into a red elliptical galaxy (Springel, Di Matteo \& Hernquist, 2005b). In Figure 4 we show as a reference the SFR, black hole accretion rate, and bolometric luminosity of model BH throughout the time period considered in this work.

The galaxies and quasars formed via this formulation for AGN feedback in galaxy merger simulations have proven consistent with observed quasar luminosity functions and lifetimes (Hopkins et al. 2006a-d, 2006a,c,d; though see Richards et al., 2006), as well as the locally observed $M_{\mathrm{BH}}-M_{\text {bulge }}$ relation (Di Matteo et al. 2005; Hopkins et al. 2007a,b; Robertson et al. 2006a,c). These simulations have also shown successes in reproducing observed X-ray, IR and

\footnotetext{
${ }^{7}$ Nuclear Coalescence is defined for the purposes of this paper as when the progenitor $\mathrm{BH}$ particles are separated by less than a smoothing length, and are thus no longer distinguishable. BH particles are included for all models in this paper, although they do not accrete in model no-winds. Coalescence could equivalently be defined as when the stellar bulges of the progenitors are no longer distinguishable, though in practice the choice of a definition for nuclear coalescence is somewhat arbitrary and makes little difference in the results. The time of coalescence is typically $\mathrm{T} \sim 1.6 \mathrm{Gyr}$, though varies slightly from model to model.
} 

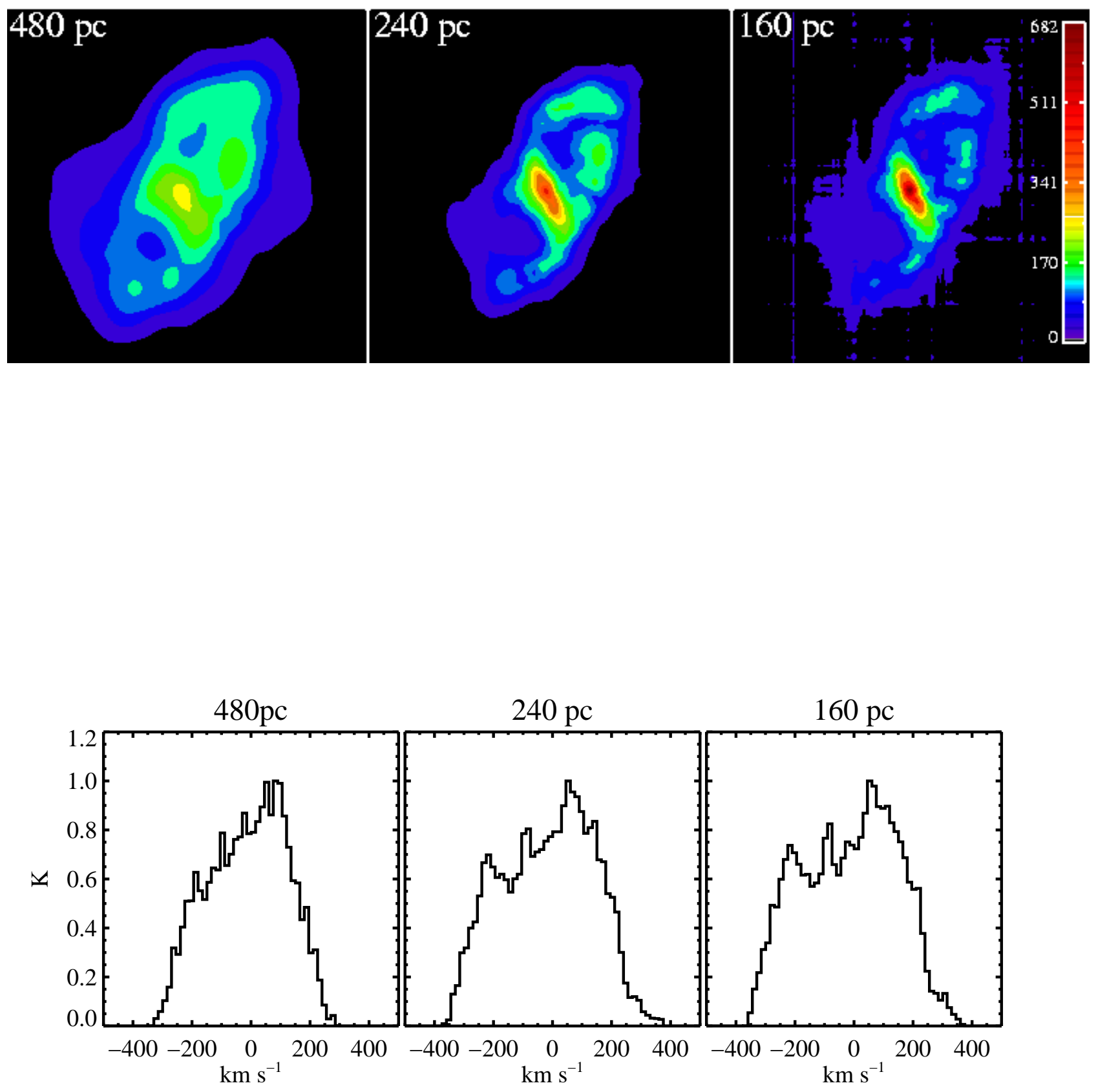

FIG. 2. - Simulated $\mathrm{CO}(\mathrm{J}=1-0)$ images and spectra for model $\mathrm{BH}$ at peak of quasar phase. Images and spectra are shown for three model resolutions. The detail in both the morphology and kinematic imprints on unresolved emission line become apparent by model resolution of $\sim 240 \mathrm{pc}$.

CO patterns characteristic of ULIRGs and quasars (Cox et al. 2006b Chakrabarti et al. 2007a,b; Narayanan et al. 2006a, respectively), the bimodal galaxy color distribution (Springel, Di Matteo \& Hernquist, 2005b; Hopkins et al. 2006b), Seyfert galaxy luminosity functions (Hopkins \& Hernquist, 2006), the kinematic structure of merger remnants (Cox et al. 2006a), the SFR-CO and SFR-HCN relation in galaxies (Narayanan et al. 2007b), and observed properties of $z \sim 6$ quasars (Li et al. 2007a,b; Narayanan et al. 2007a).

\subsection{General Wind Properties in the Simulations}

In principle, there are three mechanisms for driving winds in our major merger simulations: via shock heated gas (Cox et al. 2004, 2007), starburst-driven winds (Cox et al., 2006c, 2007) and AGN-feedback driven winds (Cox et al., 2007; Hopkins et al., 2005a-d; Springel, Di Matteo \& Hernquist
$2005 a, b)$. In practice, however, outflow rates in the simulations are dominated by winds driven by starbursts and AGN feedback. This was explicitly shown by Cox et al., (2007) who found that during the active phase, the outflow rate owing to shock heated gas was at most an order of magnitude less than that from starbursts and/or AGN. Thus for the rest of this work, we will focus primarily on the effects of winds from star formation and AGN-feedback, and rely on the model without these winds (though with shock-heated winds) as the fiducial comparative model. We note that Table 1 refers to this model as 'no-winds' though in practice winds owing to shock heated gas do exist, albeit with minimal impact on the molecular ISM.

We next review some of the broader physical effects generated by AGN feedback-driven and supernovae-driven winds. In a recent comprehensive study of galactic winds in mergers 


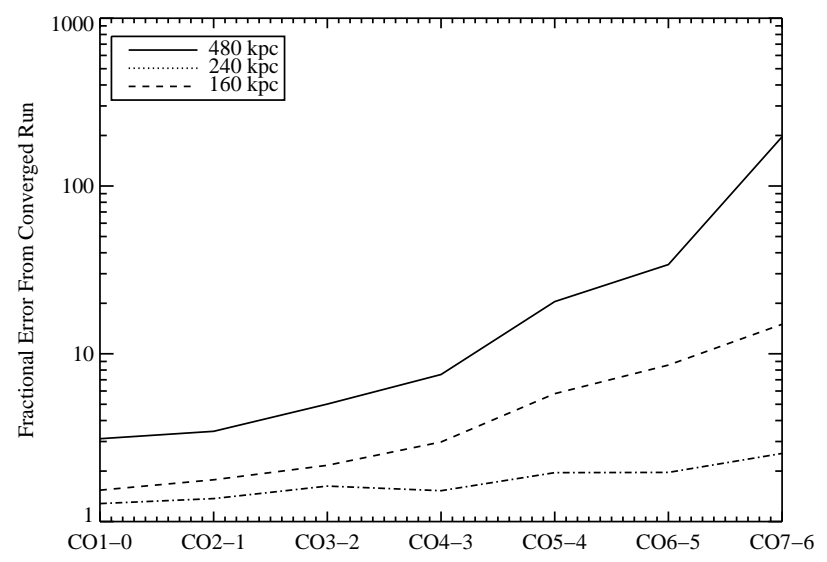

FIG. 3.- Ratio of total $\mathrm{CO}$ flux (over a variety of $\mathrm{CO}$ transitions) from highest resolution model $(\sim 120 \mathrm{pc})$ to lower resolution runs. The test snapshot is model BH during the peak of the quasar phase. The emission from higher lying transitions may be underestimated in cases of extremely poor spatial resolution.
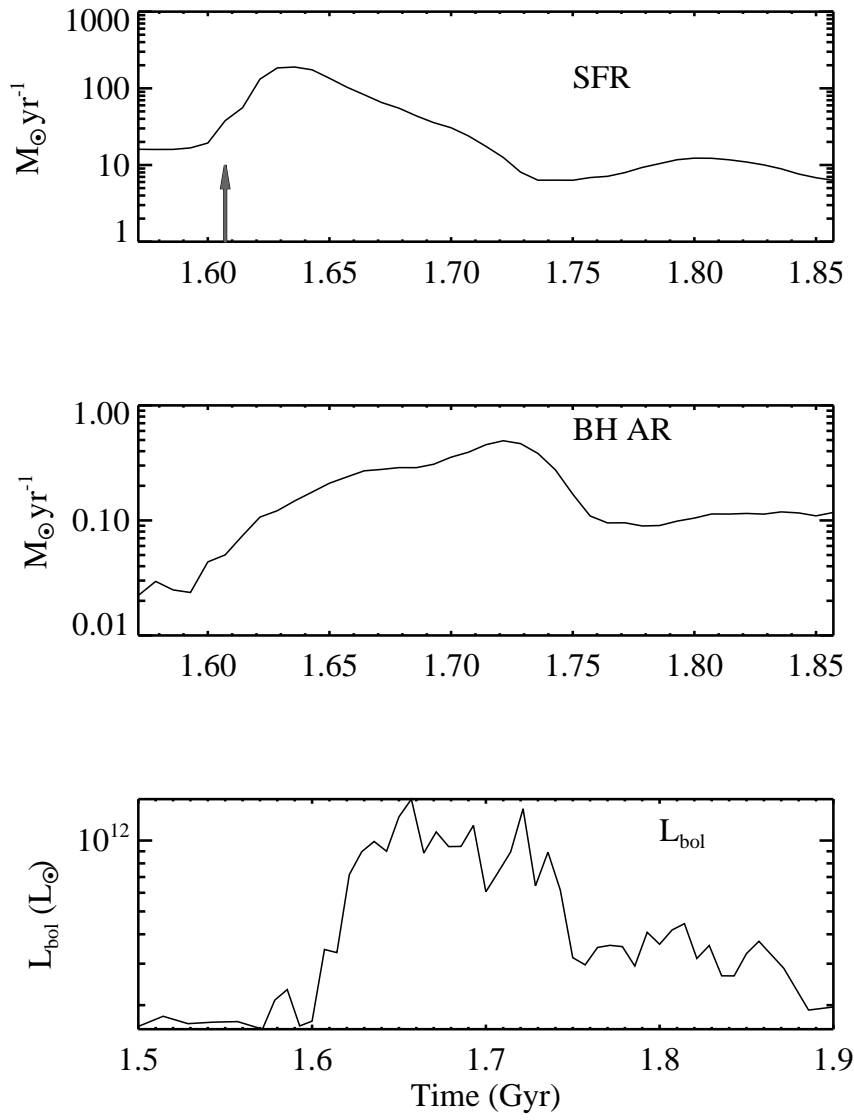

FIG. 4.- SFR, black hole accretion rate and bolometric luminosity as a function of time for model BH. The arrow in the top panel denotes the point of nuclear coalescence.

utilizing (among others) identical models to those presented here, Cox et al. (2007) analyzed the decoupled properties of starburst and AGN feedback-driven winds in galaxy merger simulations. We refer the reader to this work for a thorough analysis of these effects, and highlight here the wind properties most relevant to the discussion of this paper.

As a reference, we first show the instantaneous and inte-
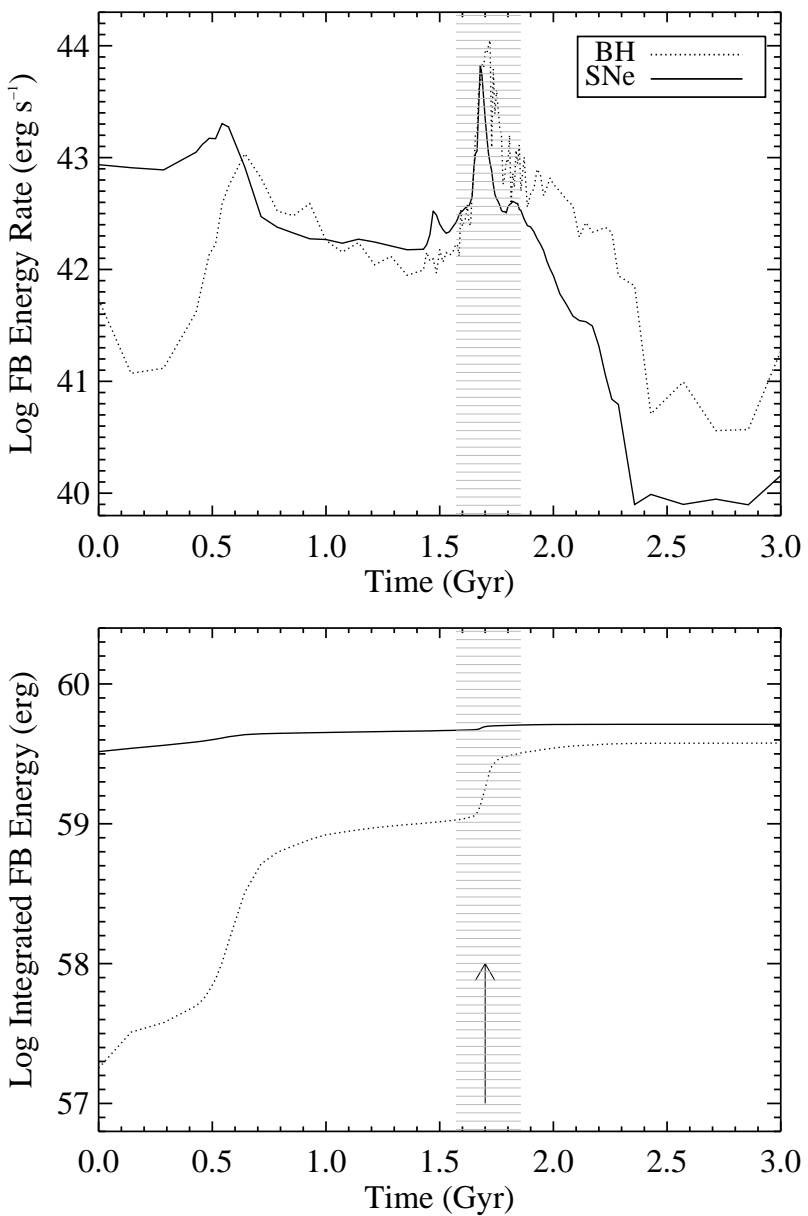

FIG. 5.- Instantaneous feedback energy rate (top) and integrated feedback energy (bottom) input into the ISM by black holes and star-formation driven winds for model sbBH. The hatched region is the $\sim 0.3 \mathrm{Gyr}$ period of peak star formation and black hole activity which will be considered for the remainder of this paper. While the integrated feedback from star formation and black holes is roughly equivalent, the star formation delivers its energy in a slow and steady manner from simulation time $\mathrm{T}=0$ spread out over much of the galaxy, in contrast to the near point explosion characteristic of the black hole winds. The arrow in the bottom panel denotes the point of nuclear coalescence. Model results first published in Cox et al. (2007).

grated feedback energies for model sbBH in Figure 5 (e.g. Equations 1 and 4, model results first published in Cox et al. 2007). We highlight the period of peak starburst and AGN activity which we will focus on for the remainder of this study, and denote as the "active phase". We focus on this period as the models exhibit their peak wind activity during this time.

The first passage of the galaxies triggers a starburst event, fueling star formation rates to $\sim 50 \mathrm{M}_{\odot} \mathrm{yr}^{-1}$, irrespective of the presence of a central black hole. At this point, starburstdriven winds begin to disrupt the central cold gas supplies, limiting to some degree (dependent on the wind efficiency and speed) the magnitude of the starburst. In the simulations presented here, during the in-spiral stage prior to nuclear coalescence, $\sim 90 \%$ of the star formation (and thus $\sim 90 \%$ of the starburst generated wind mass) occurs (Cox et al. 2007).

The growth of the central AGN and associated feedback winds develops along a markedly different story line. While the initial passage and in-spiral stage of the galaxy merger fuels central black hole growth, the majority of black hole growth occurs during the active phase highlighted in Figure 5. typically around nuclear coalescence. The exact percentage of black hole growth that occurs during the active phase is 


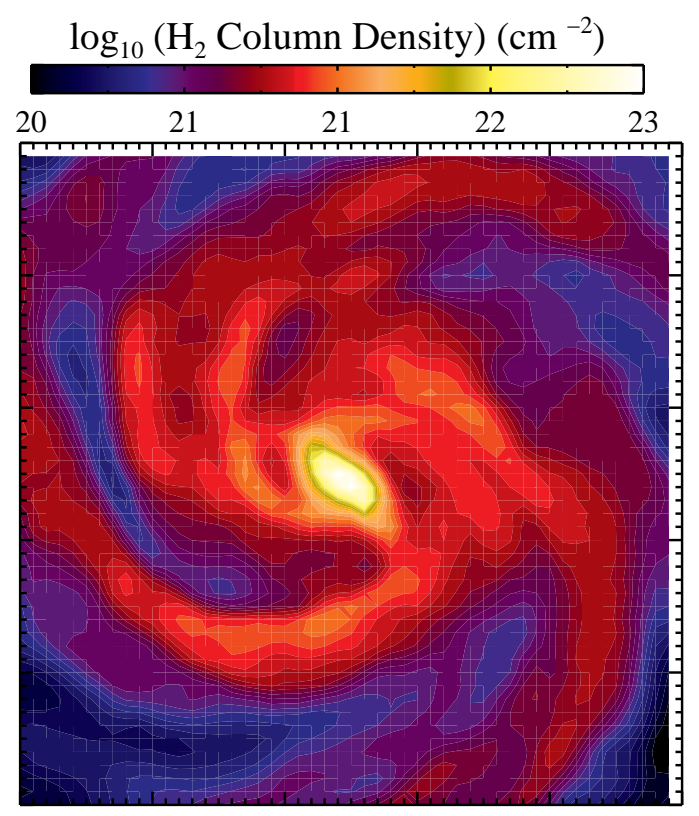

FIG. 6. $-\mathrm{H}_{2}$ column density for fiducial progenitor disk galaxy (CO contours shown in Figure 7. The panel is $12 \mathrm{kpc}$ on a side, and the scale is on top.

dependent on galaxy mass (Cox et al. 2007), but the generic trends are robust.

After the active phase, the total integrated feedback energy deposited into the ISM from star formation and black holes is roughly the same. However, the instantaneous properties are different. While the energy input from star formation winds is spread out over the entire in-spiral and active phase, the black hole offers a relatively short impulse of energy. The slow buildup of the stellar mass and relatively rapid black hole growth phase results in black holes contributing over five times the energy input to the ISM as stars during the active phase (Cox et al. 2007). This will have important consequences on the relative impact of winds on $\mathrm{CO}$ emission from starbursts and AGN. Throughout this work, we will focus on the rough peak of the active phase, specifically $\mathrm{T} \sim 1.6-1.8$ Gyr.

\section{ISOLATED DISK GALAXY}

To illustrate the generic properties of our methods, we have allowed a fiducial $40 \%$ gas fraction progenitor disk galaxy to evolve as outlined in Springel et al. (2005a). For reference, in Figure 6, we plot the $\mathrm{H}_{2}$ column density map through the model disk galaxy. In Figure 7 we present the $\mathrm{CO}(\mathrm{J}=1-0)$ through $(\mathrm{J}=10-9)$ intensity contours for comparison with observations where the intensity units are $\mathrm{K} \cdot \mathrm{km} \mathrm{s}^{-1}$ where the temperature unit is a Rayleigh-Jeans temperature.

The CO $(\mathrm{J}=1-0)$ emission traces the star forming molecular gas through the spiral arms and becomes more intense near the starbursting nuclear region. Additionally visible are individual concentrations of cold gas and star forming regions emitting as discrete pockets of $\mathrm{CO}(\mathrm{J}=1-0)$ emission through- out the spiral arms. Higher density $\mathrm{CO}$ transitions probe gas of a higher critical density. Thus the higher-lying transitions probe the higher density peaks, and become more centrally concentrated, following the density distribution in the galaxy.

At first glance, the emission contours from e.g. the $\mathrm{CO}$ $(\mathrm{J}=1-0)$ image appear to peak at higher temperatures $\left(T_{B}\right)$ than are typically recorded from observations of local star forming spirals. This effect owes to the spatial resolution of the radiative transfer models $(\sim 250 \mathrm{pc})$. When simulating more practical observing conditions, the results from our modeling are quite comparable to observations of local universe spirals. As an example, we compare our models to $50^{\prime \prime}$ observations by Scoville \& Young (1983) of CO ( $=1-0)$ emission from M51. When our model disk galaxy was scaled to be at the distance of M51 and the $\mathrm{CO}(\mathrm{J}=1-0)$ emission convolved to the same resolution as the Scoville \& Young observations, the integrated intensity in our model was comparable to the $\sim 60$ K- $\mathrm{km} \mathrm{s}^{-1}$ observed in M51 (Scoville \& Young, 1983).

In Figure 8 we present the normalized $\mathrm{CO}$ line spectral energy distribution (SED) of the model face-on disk galaxy. The CO SED represents the total integrated flux density emitted from each line and serves as a diagnostic for the excitation conditions in the molecular gas. These are plotted as the CO integrated flux density versus the upper rotational quantum number of the transition. CO SEDs are particularly useful in studies of unresolved high- $z$ sources (e.g. Narayanan et al. 2007a; Weiß et al. 2005a, 2007). We have also decomposed the CO SED by plotting the contribution to the flux from the starbursting nucleus (central kpc) and from the rest of the galaxy.

The CO flux density rises up to the $\mathrm{CO}(\mathrm{J}=5-4)$ transition, and then drops toward higher excitation lines. The intensity from the highly excited component of the $\mathrm{CO}$ gas (e.g. $\mathrm{J}>6$ ) comes primarily from the warm and dense conditions in the nuclear starburst regions, though some contribution from subthermally excited gas in the outer regions of the galaxy is visible (Narayanan et al. 2007b). This is in good agreement with observations of local starbursts. For example, in a CO SED decomposition of M82, Weiß et al. (2005b) found that the central regions peak at the $\mathrm{CO}(\mathrm{J}=6-5)$ transition whereas the total SED peaks near the $\mathrm{CO}(\mathrm{J}=4-3)$ transition. Similarly, the CO line SED from the nuclear region of NGC 253 peaks near $\mathrm{J}=7$ or $\mathrm{J}=8$ (Bayet et al. 2004; Bradford et al. 2003, 2005)

\section{CO MORPHOLOGY IN MERGERS WITH WINDS}

Our approach in combining non-LTE radiative transfer calculations with SPH simulations allows us to uniquely explore, for the first time, a variety of the decoupled effects of starburst and AGN feedback-driven winds on the molecular gas emission from galaxy mergers. In particular, a powerful tool for understanding the spatial distribution of molecular gas in local mergers is through high resolution velocity-integrated intensity contour maps. These can serve to describe the morphology and kinematics of the star-forming molecular gas, as well as characterize the nature of star-forming regions through observations of multiple excitation levels (e.g. Iono et al. 2004). Indeed, such maps have revealed, for example, the compact nature of molecular emission in mergers (Bryant \& Scoville, 1999), the double-nucleus in the central regions of Arp 220 (Sakamoto et al. 1999), and the large scale distribution of atomic and molecular gas in colliding galaxy systems (Iono, Yun \& Ho, 2005). In this section, we build on the current understanding of $\mathrm{CO}$ morphologies in interacting systems by describing the response of the $\mathrm{CO}$ morphology to starburst and 

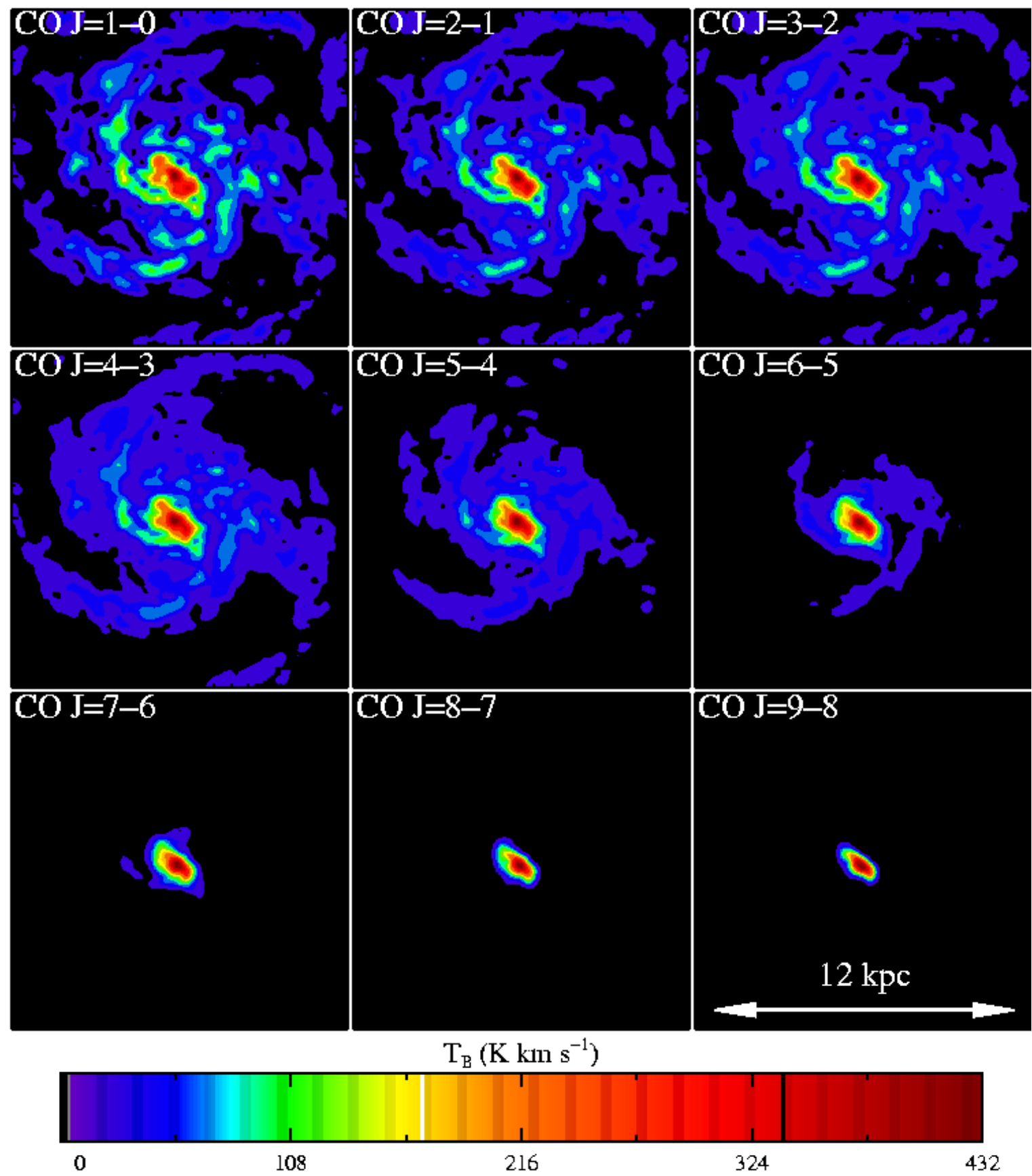

FIG. 7. - Simulated CO ( $\mathrm{J}=1-0)$ through ( $\mathrm{J}=9-8)$ emission contours for progenitor disk galaxy. While lower $\mathrm{CO}$ transitions trace the bulk of the molecular gas, higher lying transitions with relatively high critical densities probe only the nuclear star forming regions. Panels are $12 \mathrm{kpc}$ on a side, and scale on bottom is in units of $\mathrm{K}-\mathrm{km} \mathrm{s}^{-1}$.

AGN feedback-driven winds. We first discuss general properties of the $\mathrm{CO}$ morphology in mergers. We then continue with a discussion of signatures of galactic winds apparent in $\mathrm{CO}$ emission maps via outflows detectable in the CO morphology. We conclude the section with a brief discussion of secondary effects of galactic winds in affecting the spatial extent of $\mathrm{CO}$ gas.

\subsection{General Properties of CO Morphology}

In Figure 9, we show the evolution of the $\mathrm{CO}(\mathrm{J}=1-0)$ emission during a major merger of two disk galaxies simulated in each of our models, regardless of wind parameter choice. The images in Figure 9 start after the first passage of the galaxies, when they are approaching for their final coalescence. The tidal interaction of the first passing of the galaxies causes 'streamer' gas to emit in the intergalactic space between the two galaxies. The $\mathrm{CO}$ morphologies are disturbed by the first passage itself, and the $\mathrm{CO}$ emission is extended in nature until the nuclei have fully merged. The first passage of the galaxies induces a burst of star formation, peaking in the nuclear regions of each galaxy. This is reflected in the luminous $\mathrm{CO}$ emission at the center of each galaxy.

Because the $\mathrm{CO}(\mathrm{J}=1-0)$ can be thermalized at relatively low 


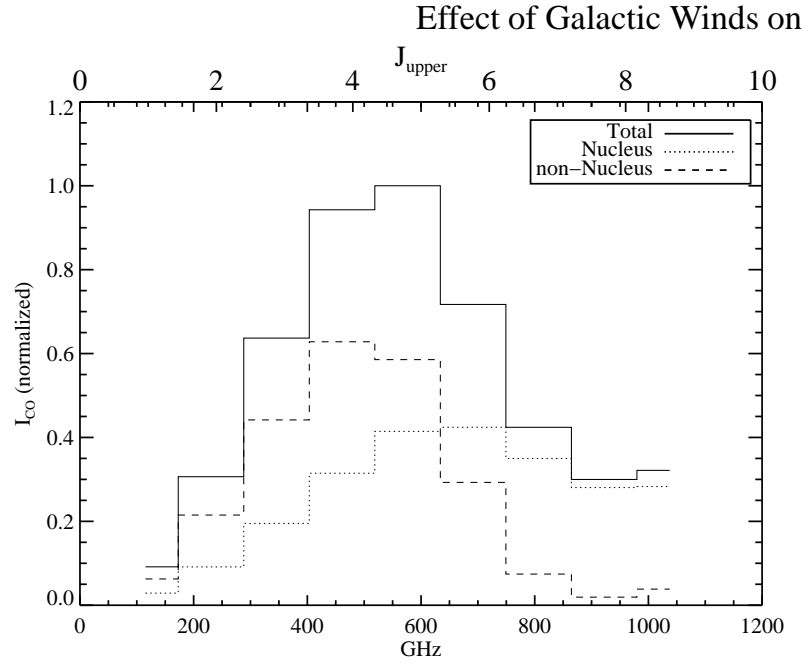

FIG. 8.- Normalized CO SED of representative progenitor disk galaxy. The CO SED is decomposed into contribution from the nuclear (central kpc) region and the rest of the galaxy. The CO SED shows the relative flux density for different $\mathrm{CO}$ transitions, and plots the frequency of the transition on the bottom axis and upper $\mathrm{J}$ state on the top axis. The peak in the total CO SED as well as that just from the starbursting nucleus is consistent with results derived from local starburst galaxies, supporting our methodology for deriving $\mathrm{CO}$ excitation patterns in galaxy simulations (Bradford et al. 2005; Weiß et al. 2005b).

as a good tracer for total molecular gas content. Both diffuse envelopes of clouds as well as dense cores can emit $\mathrm{CO}(\mathrm{J}=1$ 0) flux. Conversely, emission from higher lying lines of $\mathrm{CO}$, and high dipole moment molecules (e.g. HCO+, HCN, CS) arises primarily from dense cloud cores. Thus, higher lying $\mathrm{CO}$ lines (e.g. $\mathrm{CO} \mathrm{J}=3-2$ ) from the merger broadly follow the distribution of the $\mathrm{CO}(\mathrm{J}=1-0)$ line, but remain more compact in nature, generally emitting only from the dense starbursting nucleus. This was explicitly seen in the disk galaxy in Figure 7 as well.

The nuclear CO intensity peaks soon after the progenitor galaxies lose individual identity, and there is only one surface brightness peak. During this time, stars formed in the mergerinduced starburst heat up the molecular gas such that collisions readily excite the $\mathrm{CO}$ in the warm $(\mathrm{T} \sim 30 \mathrm{~K})$ and dense $\left(n \sim 10^{5} \mathrm{~cm}^{-3}\right.$ ) gas. The columns are still quite large through the nucleus as the starburst has not had time to consume the available molecular gas.

While the nuclear CO intensity peaks near coalescence, the globally averaged velocity-integrated $\mathrm{CO}$ intensity across all transitions drops through the evolution of the merger. This can be attributed to global consumption of cold molecular gas by star formation over time. This result is consistent with observations by Gao \& Solomon (1999) who found from CO $(\mathrm{J}=1-0)$ emission that the total molecular gas content of a merger decreases as a function of projected nuclear separation in galaxy mergers.

\subsection{Molecular Outflows}

Starburst and AGN feedback-driven winds can leave an imprint on the molecular line emission via large scale molecular outflows seen in the $\mathrm{CO}$ morphology. These winds are seen to entrain large masses of molecular gas in outflows which appear prominently as a secondary surface brightness peak in velocity-integrated emission contour maps (Narayanan et al. 2006a).

In this section, we discuss the properties of the $\mathrm{CO}$ mor- ble via $\mathrm{CO}(\mathrm{J}=1-0)$ emission maps. For the purposes of understanding general trends of detectable outflows in this section, we will find it convenient to define a detectable outflow as any $\mathrm{CO}$ emission peak at least $50 \%$ of the nuclear emission maximum, with a projected distance at least $1.5 \mathrm{kpc}$ away from the galactic nucleus. The molecular outflows are defined only after nuclear coalescence, and thus also follow the requirement that the $\mathrm{CO}$ emission peak be separate from the stellar surface density peak.

Before continuing, we note that the simulations chosen here are not extreme in any way (Figure 4). The peak B-band luminosity during the quasar phase is $\sim 10^{11} \mathrm{~L} \odot$. Similarly, the peak IR luminosity is $\sim 10^{12} \mathrm{~L}_{\odot}$, allowing the galaxy to be just visible as a ULIRG. More massive mergers (e.g. Cox et al. 2007), and, as will be discussed in $\$ 4.2 .3$ more coplanar mergers will drive stronger winds, leaving the simulations in this section representative of lower limits.

\subsubsection{Outflow General Properties}

Here, we discuss the general properties of both starburst and AGN feedback-driven outflows which appear in $\mathrm{CO}$ emission maps. While there are differences in some specific properties of the outflows based on the type or strength of the driving wind, we defer those comparisons to later subsections and focus this subsection on the outflow properties shared by all the simulations presented here.

In Figure 10, we show an example of molecular gas entrained in the outflow from model BH (AGN winds only; outflow first described in Narayanan et al. 2006a). The cold gas in the outflow is not completely ablated by starburst and AGN heating. While the $\mathrm{CO}$ emission from cold gas in the outflows closest to the nucleus is faint owing to lower columns of cold molecular gas, clouds which remain more deeply embedded in the outflow continue to exhibit active star formation. During short ( $\sim 5$ Myr) time intervals, the $\mathrm{CO}(\mathrm{J}=1-0)$ velocityintegrated intensity from galactic outflows can be seen to be comparable (within a factor of $\sim 2$ ) to the galaxy's nuclear emission.

The detectable outflows typically correspond to entrainments of mass $\sim 10^{8}-10^{9} \mathrm{M}_{\odot}$, with peak $\mathrm{H}_{2}$ column of $\sim 10^{22}$ $10^{23} \mathrm{~cm}^{-2}$ (within the constraints of our definition of a detectable outflow, $\S 4.2$. The more massive outflows in this mass range tend to be seen closer to the point of nuclear coalescence, with a trend toward decreasing outflow mass as the merger progresses. This owes to more loosely bound gas near the time of coalescence, as well as the feedback energy rate being near its peak (Figure 5). These outflow masses are typically $5-15 \%$ of the total molecular gas mass. In the mergers $\mathrm{e}, \mathrm{sb}$, and $\mathrm{sbBH}$, with random disk orientation angles ${ }^{8}$, the outflows do not occur in any preferred direction. As we will discuss later, this is not the case for the coplanar model.

The entrained clouds can be relatively excited, allowing outflows to be imageable at higher-lying $\mathrm{CO}$ transitions (e.g. $\mathrm{J} \gtrsim 4$ ). In Figure 11, we show the outflow from the first panel of Figure 10 in $\mathrm{CO}$ transitions $\mathrm{J}=1-0, \mathrm{~J}=3-2$ and $\mathrm{J}=4-3$. The latter two transitions have a relatively high critical density of $\gtrsim 10^{4} \mathrm{~cm}^{-3}$ meaning they are typically thermalized only in the dense cores of GMCs, and may rely on radiative pumping for excitation in other parts of the ISM. The warm and dense gas heated by active star formation in the deeply embedded clouds in the outflows collisionally excite the $\mathrm{CO}$ gas into higher levels. In fact, the $\mathrm{CO}$ flux density in the outflow peaks at $\mathrm{J}=4$, 


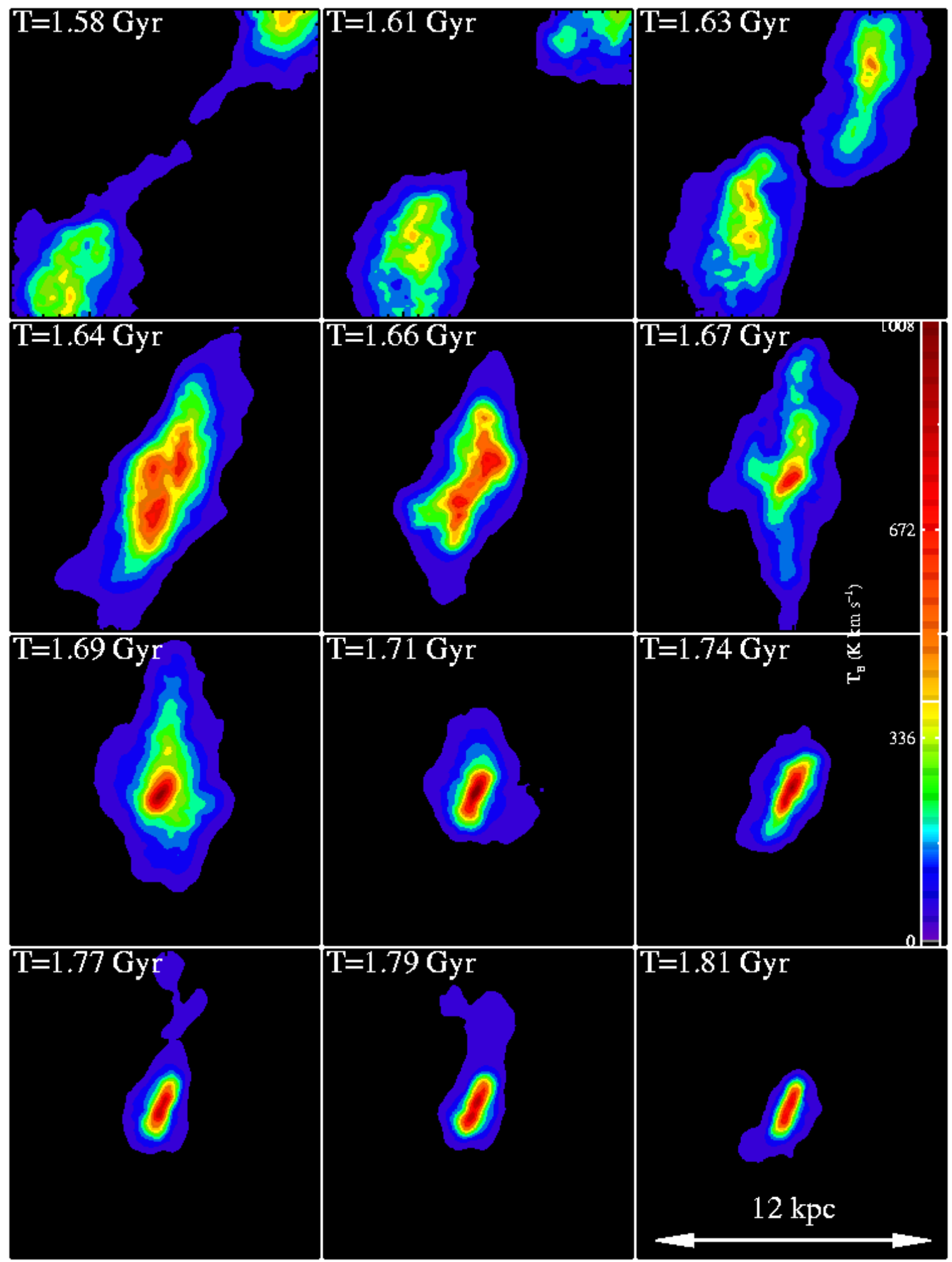

FIG. 9.- Contours of velocity-integrated $\mathrm{CO}(\mathrm{J}=1-0)$ emission at various snapshots through evolution of merger model sbBH, with both starburst and AGN feedback-driven winds. The results discussed here are generic for all wind models. Upon nuclear coalescence ( $\mathrm{T}=1.7 \mathrm{Gyr}$ ) a burst of star formation drives the $\mathrm{CO}$ luminosity to near peak values. The CO luminosity then fades as star formation consumes the available molecular gas. Units are $\mathrm{K}-\mathrm{km} \mathrm{s}^{-1}$ (scale at bottom), and each panel is $12 \mathrm{kpc}$ on a side. 
consistent with actively star-forming gas and multi-line measurements of excitation patterns in starburst-driven outflow of M82 (Walter, Weiß \& Scoville, 2002). It should be noted that the relative visible lifetimes of outflows in higher-lying transitions may be much smaller than at $\mathrm{CO}(\mathrm{J}=1-0)$. For example, the outflow presented in Figure 10 appears as a unique secondary surface brightness peak for $\sim 35 \mathrm{Myr}$ at $\mathrm{CO}(\mathrm{J}=1-0)$. In the $\mathrm{CO}(\mathrm{J}=3-2)$ transition, the same outflow is distinguishable from the background for only $\sim 20 \mathrm{Myr}$.

\subsubsection{Relative Role of AGN and Starbursts in Powering Detectable CO Outflows}

As an illustrative point demonstrating the relative contributions of starburst and AGN winds in driving detectable molecular outflows, in Figure 12 we plot three snapshots during the period of nuclear coalescence for models no-winds, $\mathrm{BH}, \mathrm{sb}$, and $\mathrm{sbBH}$ (thus varying only the form of winds included, and not geometry of the merging galaxies). The wind-dependent morphological differences in the models are typically minimal near the point of nuclear coalescence, and thus the outflows imaged at this time serve as a reasonable comparison for the capacity of different wind models to drive outflows. Here, loosely bound gas near the inner regions is seen to be entrained in nuclear galactic winds. For reference, these outflows are the same as those shown in Figures 10 and 11

Model BH is able to drive strong outflows (4th row, Figure 12) with properties described generally in $\$$ 4.2.1. The black hole feedback is tightly associated with the accretion rate, and thus the AGN wind is produced over a very short period of time and peaks soon after nuclear coalescence. Relative to the evolution of the galaxy, the AGN wind generically acts like a point explosion, and the bulk of the energy is imparted to the ISM nearly instantaneously. Indeed, Hopkins et al. (2006c) and Hopkins \& Hernquist (2006) have shown explicitly that these AGN-driven winds are well-characterized by generalized Sedov-Taylor solutions for point explosions. The outflows in model BH are primarily seen soon after the major merger, when the gas is still highly dynamical and loosely bound. They are also seen near the height of quasar activity, when the AGN feedback-driven winds are strongest.

The starburst driven winds are also able to power outflows (2nd row, Figure 12), albeit less strongly than in the AGNwind models; consequently, these outflows are detectable via emission mapping for shorter periods of time. The typical lifetime of an outflow such that it is detectable in $\mathrm{CO}(\mathrm{J}=1$ 0 ) in the sb simulation (with only starburst winds) is $\sim 20$ $25 \mathrm{Myr}$ (compared with $\sim 35 \mathrm{Myr}$ in the AGN only model). On average, $3-5 \times 10^{8} \mathrm{M}_{\odot}$ are entrained, although outflows as small as $\sim 10^{7} \mathrm{M}_{\odot}$ are seen to occur, consistent with observations of starburst driven outflows by Sakamoto et al. (2006) and Walter et al. (2002). It should be noted that these values are derived within the constraints of the parameters chosen for our starburst winds $\left(\eta=0.5, v_{w}=837 \mathrm{~km} \mathrm{~s}^{-1} ; \S 2.1\right)$. In principle, a more efficient wind might increase the mass outflow rate, but, as discussed in Cox et al. (2007), such parameter choices were found to quench merger-induced starbursts, and are thus probably not representative of e.g. ULIRGs.

The relative weakness of starburst-driven outflows compared to the AGN only model is a direct consequence of the lower peak energy input from the starburst-driven winds, and hence lower power. During the active phase, the feedback from black holes inputs more than five times the energy as verting accreted mass into thermal energy than star formation. For example, by utilizing Equations (1) and (4), Cox et al. (2007) noted that the relative efficiency of black holes to star formation in converting gas mass to energy deposited into the ISM was of order $\sim 640$ (see, also, Lidz et al. 2007). Second, during the active phase, the relative black hole growth rate is much larger than the concomitant SFR. During this time, while the $\mathrm{BH}$ grows by a factor of $\sim 4$, only $\sim 8 \%$ of the final stellar mass is formed (Cox et al. 2007). In contrast, $~ 90 \%$ of the stellar mass (and consequently, stellar winds) is produced during the in-spiral stage, prior to the active phase.

The effect of including both starburst and AGN winds is demonstrated in the third row of Figure 12. The outflows produced are more massive and longer lived than the starburstonly wind models, but less so than the AGN-only wind models. The greater capacity to drive outflows over the starburst winds only model ( $\mathrm{sb}$ ) can be attributed to the extra energy input given by the accreting black hole(s). However, the outflows in Figure 12 are seen to be less powerful than those in the black hole only model. This effect owes to the starburst winds that occur prior to nuclear coalescence. As shown explicitly by Cox et al. (2007), starburst winds lower the amount of rotationally supported gas early during the in-spiral stage, which limits the amount of gas available for immediate funneling into the central nucleus via tidal torquing (Hernquist, 1989; Mihos \& Hernquist, 1994b, 1996). This gas does eventually rain into the nuclear potential, fueling black hole growth and producing a black hole with the same mass as in the AGN wind only model (BH) and a galaxy that lies on the $\mathrm{M}_{\mathrm{BH}^{-}} \sigma_{\mathrm{v}}$ line (Cox et al. 2007; Di Matteo, Springel \& Hernquist, 2005; Robertson et al. 2006a), as well as the black hole fundamental plane (Hopkins et al. 2007a,b). However, the black hole growth is integrated over significantly longer time, and thus the impact of the associated winds on molecular gas emission is diminished from the AGN-only model.

The relative effects of AGN feedback and starburst-driven winds on powering molecular outflows can be summarized in Figure 13, where we plot the mass of detectable outflows as a function of time for models $\mathrm{BH}, \mathrm{sb}$ and $\mathrm{sbBH}$. The masses of gas each model is able to drive in an outflow is comparable to within a factor of $\sim 2-3$. AGN feedback-driven winds drive outflows clustered primarily around nuclear coalescence and the peak period of black hole accretion. In comparison, the models with starburst driven winds ( $\mathrm{sb}$ and $\mathrm{sbBH}$ ) power detectable outflows for relatively shorter periods.

\subsubsection{Merger Orbit Dependence}

The strength of outflows in merger models depends on the initial orientation of the merging galaxies. In models where the disks are aligned, strong tidal torquing can cause a rapid inflow of gas thus providing a sudden burst of AGN feedback energy injected into the ISM. Alternatively, geometries such as those in the $\mathrm{BH}$ and no-winds models by comparison experience less tidal torquing on the gas, causing the major black hole accretion phase to be longer lived. In order to investigate the dependence of $\mathrm{CO}$ outflows on merger orbit, we have run simulation co-BH (Table 1 in which the progenitor galaxies are coplanar with the orbital plane $\left(\phi_{1}=\theta_{1}=\phi_{2}=\theta_{2}=0\right)$. The stronger tidal torquing in the coplanar configuration results in a more efficient blow-out of material. The $\mathrm{H}_{2}$ gas in the galaxy remains confined in a thin configuration in the plane of the merger, consistent with previous simulations which 

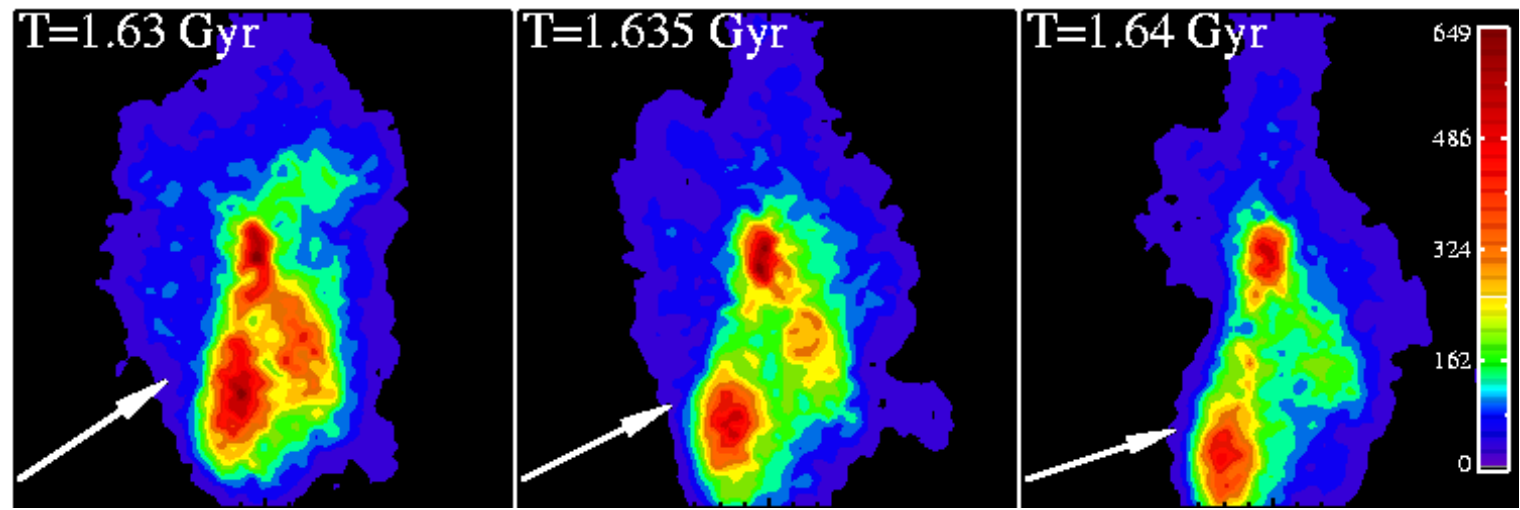

FIG. 10.- Velocity-integrated CO (J=1-0) emission map of detectable molecular outflows seen in the model with black hole winds only (see also Narayanan et al. 2006a). Thermally driven winds associated with black hole growth can entrain large masses $\left(\sim 10^{8}-10^{9} \mathrm{M}_{\odot}\right)$ of molecular gas, resulting in directly detectable outflows. For reference, nuclear coalescence is at $\mathrm{T}=1.6 \mathrm{Gyr}$. The units are $\mathrm{K}-\mathrm{km} \mathrm{s}^{-1}$ (scale on right), and the panels are $12 \mathrm{kpc}$ each.
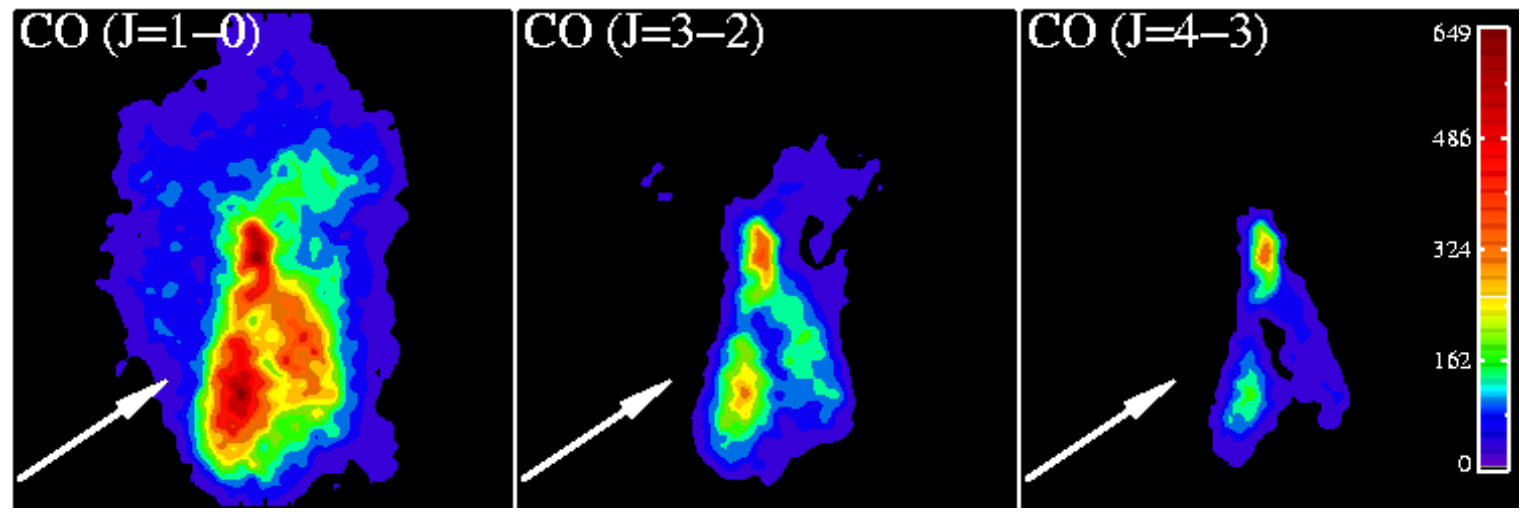

FIG. 11. - First panel of Figure 10 in commonly observed CO transitions $\mathrm{J}=1-0, \mathrm{~J}=3-2$, and $\mathrm{J}=4-3$. The massive and dense outflows entrain numerous GMCs, and continue to actively form stars. Dense cores in entrained GMCs emit strongly in higher-lying CO transitions, and can be imaged in J=3-2 and J=4-3, at submillimeter wavelengths. The outflow lifetime typically drops with transition as the outflows become more diffuse further from the nuclear regions (see text). For reference, nuclear coalescence is at $\mathrm{T}=1.6 \mathrm{Gyr}$. The intensity scale in $\mathrm{K}-\mathrm{km} \mathrm{s}^{-1}$, and the scale is on the right. Each panel is $12 \mathrm{kpc}$ on a side.

In Figure 14, we show a typical outflow from the coplanar model. Similar to the other models in Table 1, the primary molecular outflows occur at or near the point of nuclear coalescence. Unlike models sb/sbBH/BH, which showed no preferred direction for outflows, the outflows in coplanar model co-BH are seen to occur almost exclusively in the plane of the merger with an expanding shell-like structure. ${ }^{9}$ The fraction of time in our simulations outflows are visible is dramatically increased as well in this merger model. In the $\mathrm{sb} / \mathrm{sbBH} / \mathrm{BH}$ models, the outflows typically involve loosely bound gas entrained in AGN winds which fall back into the nuclear potential, tending the emission toward a centrally concentrated morphology. Conversely, the outflows in the coplanar model appear to continue expanding within a $6 \mathrm{kpc}$ (radius) region, resulting in an increasing half light radius throughout the evolution of the post-merger galaxy. The continued presence of detectable outflows in the coplanar model is seen explicitly in Figure 15, where we show the mass of detectable outflows for models $\mathrm{BH}$ and co-BH (thus varying only the orientation angle) as a function of time.

The outflows are most dramatic for face on viewing angles as the outflows are preferentially within the plane of the

9 We caution that the outflows seen here are unrelated to the "shells" and "ripples" seen around many merger remnants and ordinary ellipticals. These features are stellar-dynamical in origin and are caused by "phase-wrapping" of stars (e.g. Quinn 1984; Hernquist \& Quinn 1987) as tidal debris falls into the potential well of the remnant (Hernquist \& Spergel 1992). Indeed, gas merger. While at some inclined angles outflows continue to be visible, broadly, the more edge-on the viewing angle becomes, the more difficult outflows become to detect against nuclear emission. That said, outflows may be preferentially detectable along these sightlines via spectral line signatures which we will discuss further in $\S 5$. Within the plane of the merger, outflows can be viewed in outflow, or falling back into the nucleus throughout the entire $\sim 200$ Myr peak of the active phase investigated here.

The peak black hole accretion rate and integrated energy deposited into the ISM are similar for both the coplanar model and model BH. Consequently, the typical physical conditions of the AGN feedback-driven outflows are roughly the same in the two simulations. In the coplanar model discussed in this section, the typical mass of an outflow is a few $\times 10^{8} \mathrm{M}_{\odot}$, similar to the outflows observed in model $\mathrm{BH}$ which had a more generic inclination angle for the progenitor galaxies.

\subsection{CO Half-Light Radius}

Galactic winds can play a role in the spatial extent of $\mathrm{CO}$ emission in galaxy mergers. After nuclear coalescence, the $\mathrm{CO}(\mathrm{J}=1-0)$ emission in the simulation without winds (model no-winds) is seen to be more spatially extended than the emission from mergers with winds by a factor of $\sim 2$. The CO $(\mathrm{J}=1-0)$ emission from model no-winds has a typical halflight radius of 1-2 kpc (Figure 16, top panel). The $\mathrm{CO}$ emission 


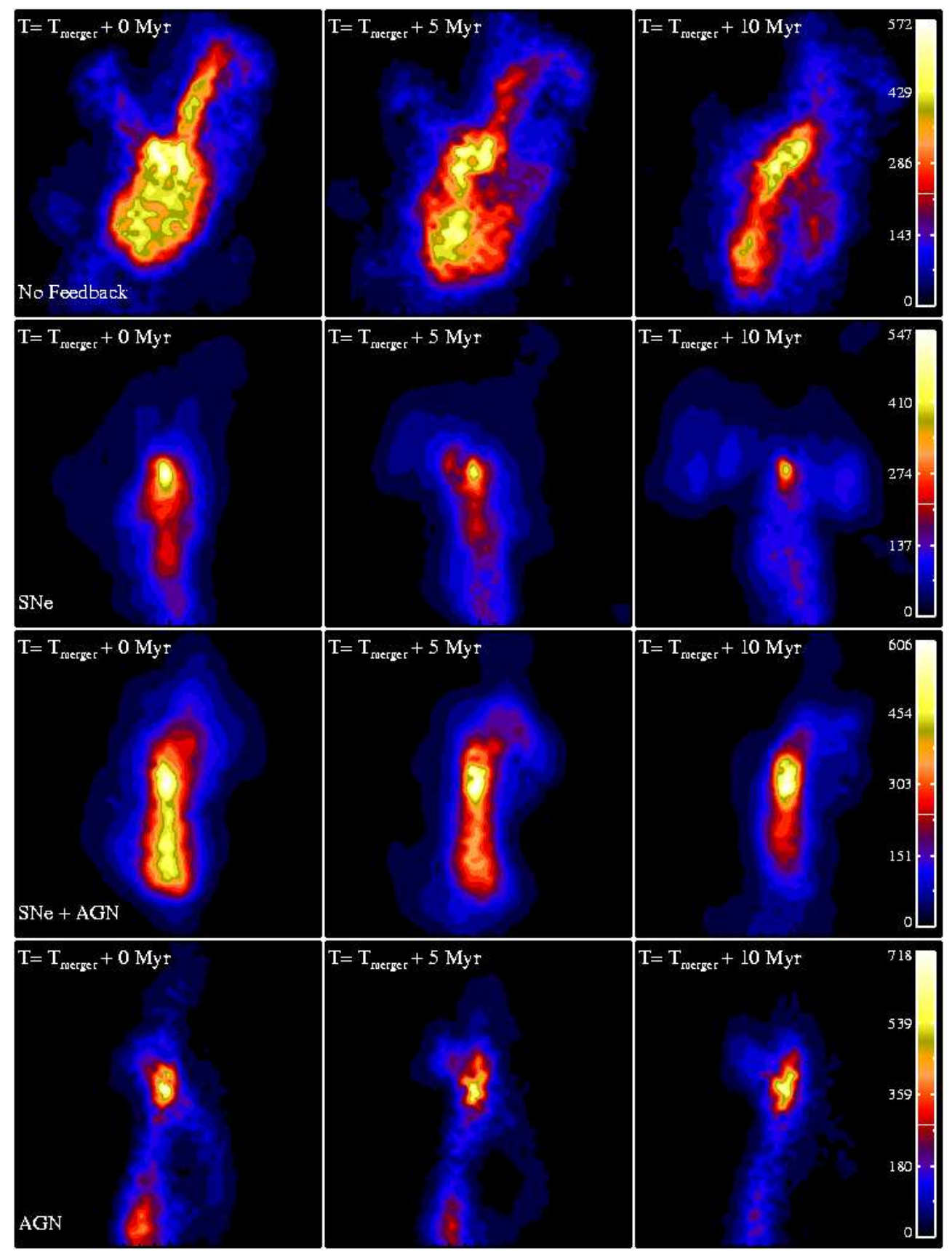

FIG. 12.- $\mathrm{CO}(\mathrm{J}=1-0)$ emission contours in snapshots of models BH, no-winds, sb and sbBH (thus varying only wind models) near nuclear coalescence. The first snapshot in each row is the point of nuclear coalescence, and the timestamps at the top left refer to the length of time after coalescence. Loosely bound gas soon after the merger is visible in the nuclear regions of the model without winds (no-winds, top row). As the strength of the winds increases (second through fourth rows), this gas is entrained and can be imaged in outflow. The wind with the strongest instantaneous impact is the AGN-only simulation (model BH) as the presence of starburst winds can slow accretion onto the central black hole. Thus, the outflows in the AGN-only wind model are the most dramatic. Each panel is $12 \mathrm{kpc}$, and the contours are in units of $\mathrm{K}-\mathrm{km} \mathrm{s}^{-1}$. For clarity in the images, we have plotted each model on its own color scale, though note that the scales are roughly similar (scale on the right of each row).

molecular gas as fuel.

The predicted lack of extended $\mathrm{CO}(\mathrm{J}=1-0)$ emission in galaxy mergers with winds is consistent with observations. In a high resolution $\mathrm{CO}$ survey of five mergers, Bryant \& Scoville (1999) found the primary CO emission to be compact and concentrated within a $300-400$ pc radius of the nucleus in three sources, and within $1.6 \mathrm{kpc}$ in all of the objects in the sample. Moreover, high resolution observations and radiative transfer modeling of local mergers have confirmed the compact nature of $\mathrm{CO}$ emission in mergers (Downes \& and/or starbursts may help to explain this.

In the models with winds, as the galaxies merge, the sudden inflow of gas into the nucleus rapidly drives up the nuclear star formation rate, as well as the accretion rate of the black hole(s). The feedback associated with star formation and the central AGN is effective at heating the gas in the nuclear regions, and dispersing lower density $\mathrm{CO}(\mathrm{J}=1-0)$ gas, leaving behind only a dense core of cool gas in the central regions in the winds models. The average $\mathrm{CO}(\mathrm{J}=1-0)$ half light radius after the nuclei have coalesced for the models with SNe and/or 


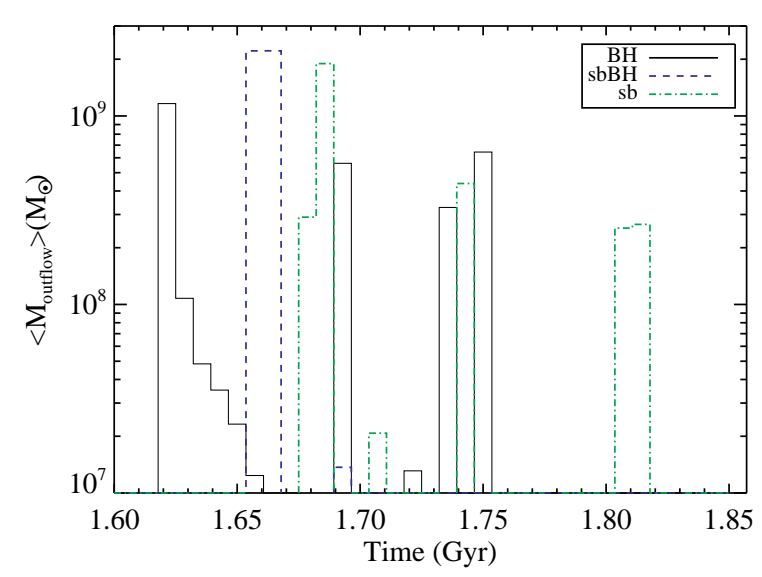

FIG. 13.- Masses of detectable outflows as a function of time for model $\mathrm{BH}$, averaged over three orthogonal sightlines for models $\mathrm{BH}, \mathrm{sb}$ and $\mathrm{sbBH}$. The masses of a typical detectable outflow are comparable between models. The AGN-only model drives outflows preferentially near nuclear coalescence, and for longer integrated periods of time. Comparatively, the simulations with starburst winds generally drive outflows for shorter periods. For reference, the times of nuclear coalescence are $\mathrm{T}=1.6 \mathrm{Gyr}$ for model $\mathrm{BH}$ and $\mathrm{T}=1.7$ Gyr for models sbBH and sb.

showed that the major $\mathrm{CO}(\mathrm{J}=1-0)$ intensity for the model with $\mathrm{SNe}$ and $\mathrm{AGN}$ winds originates in a compact region, in agreement with observations of local ULIRGs (Bryant \& Scoville, 1999; Downes \& Solomon, 1998).

In principle, these model results can be directly tested through observations of higher lying $\mathrm{CO}$ lines from local galaxies. The middle and bottom panels of Figure 16 show the halflight radius as a function of time for models $\mathrm{BH}$ and nowinds for the $\mathrm{CO}(\mathrm{J}=3-2)$ and $\mathrm{CO}(\mathrm{J}=7-6)$ transitions. These transitions probe denser gas than $\mathrm{CO}(\mathrm{J}=1-0)$, and have a critical density of $\gtrsim 10^{4}-10^{5} \mathrm{~cm}^{-3}$. Figure 16 shows that the emission from the model with no winds is stratified such that tracers of dense gas [e.g. $\mathrm{CO}(\mathrm{J}=7-6)]$ are more compact by nearly an order of magnitude than tracers of lower density gas [e.g. $\mathrm{CO}(\mathrm{J}=1-0)]$. In Figure 17 we show the mean half light radius for each model plotted as a function of $\mathrm{CO}$ transition. Here, we have averaged the half light radius after nuclear coalescence in each simulation. The $\mathrm{CO}$ emission in the model without winds becomes more compact in higher lying transitions (e.g. those with higher critical densities). In the models with $\mathrm{SNe}$ and/or AGN winds, the $\mathrm{CO}$ emission shows a similar behavior, but the halflight radius for the lowest transitions is about a factor of two smaller than in the models without winds.

With these predictions, we caution that specific values quoted here are dependent on a number of assumptions included in parameter choices (such as, for example, the specific multi-phase ISM breakdown [e.g. Springel \& Hernquist, 2003] or constant CO abundances), and thus the normalization of the plots presented are to be taken within those confines. However, the general trends are likely to be more robust. In this sense, the relatively shallow slope in half-light radius versus transition (Figure 17) remains a valid observational test for the role of galactic winds in shaping observed CO spatial extents. Finally, it is important to note that the weak trend in average halflight radius as a function of transition in Figure 17 is an ensemble-averaged trend over three orthogonal sightlines and numerous points in the post- ter the progenitors have coalesced - in other words, after the merger has only a single nuclear emission peak in the $\mathrm{CO}$ morphology.

\section{SIGNATURES OF WINDS IN CO LINE PROFILES}

\subsection{Overview}

Generically, when the nuclei of the progenitor galaxies are still resolvable, prior to coalescence, the unresolved $\mathrm{CO}$ emission line exhibits multiple emission peaks corresponding to the nuclear regions of each galaxy (e.g. Greve \& SommerLarsen, 2006). When the molecular gas in galaxy mergers becomes dynamically relaxed, the $\mathrm{CO}$ emission line is reasonably well represented by a single broad Gaussian. Alongside this general trend, at various times throughout the evolution of the merger, secondary (and potentially, though less commonly, tertiary and quaternary) peaks can be seen superposed on the broad CO Gaussian-like line. In Figure 18, we show a representative evolution of $\mathrm{CO}$ spectral lines, and how they correspond to the $\mathrm{CO}$ morphology. These secondary peaks superposed on the broad Gaussian originate in massive $\mathrm{CO}$ gas clumps moving along the line of sight. In Figure 18, the secondary peak in the third panel represents emission from the outflowing clump visible in the corresponding $\mathrm{CO}$ contour image.

While these superposed secondary emission peaks are a general feature of all models in our simulations, the physical origin of these peaks is dependent on the strength of the winds included in a given merger model. In particular, secondary emission peaks with velocities greater than the circular velocity typically represent loosely bound gas which has not virialized (peaks at velocities lower than the circular velocity may also be loosely bound gas, though they may also represent gas in rotational support; for brevity we hereafter refer to secondary peaks at velocities greater than the circular velocity as 'high velocity peaks'). As we will show more quantitatively, in models which include winds, this high velocity gas is typically entrained in outflow during periods of activity (e.g. elevated star formation rate or black hole growth), and thus give rise to high velocity peaks in the emission line representative of outflowing gas. This is analogous to the situation seen in the CO morphologies presented in Figure 12 when loosely bound gas simply fell into the central potential in the model without winds, but was entrained in outflow in models with winds (here, models sb, sbBH and $\mathrm{BH}$ ). This effect of winds on $\mathrm{CO}$ emission profiles was first shown explicitly in similar calculations by Narayanan et al. (2006a). This work demonstrated that once the galaxies merged, large columns of gas outflowing along the line of sight can leave their imprint on the broad single-Gaussian emission line characteristic of galactic $\mathrm{CO}$ emission by introducing an additional superposed emission peak, red or blueshifted at the line of sight velocity of the outflow.

The relative significance of high velocity peaks compared to the main emission line decreases as the gas virializes in the merger. To illustrate this, in Figure 19 we plot the evolution of the goodness of fit (taken here as the median of the square of the residuals) for a single Gaussian fit to the emission lines from model $\mathrm{BH}$, although the results are common across all models. The impact of winds in driving high velocity peaks decreases as the merger progresses.

In our models, high velocity peaks in $\mathrm{CO}$ emission lines are typically representative of clumps of gas moving along 

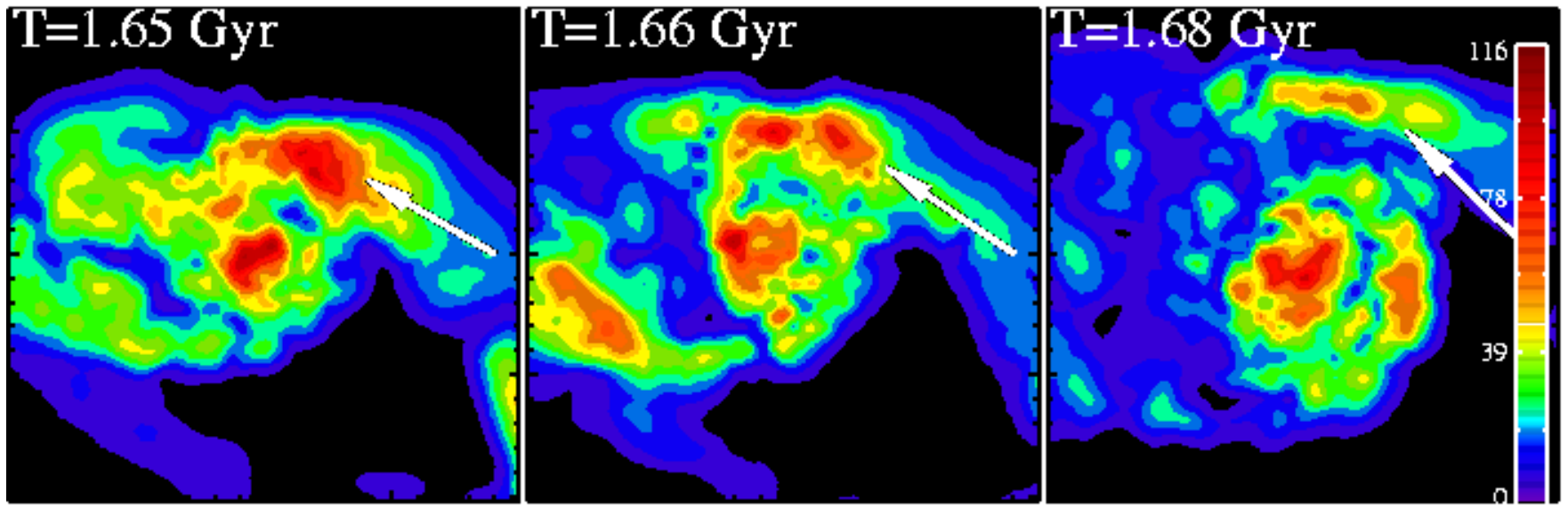

FIG. 14.- Coplanar merger simulation, co-BH, soon after nuclear coalescence. The molecular outflows occur preferentially in the plane of the merger in a shell-like structure. An emergent outflow is visible near the top of the nuclear region, as well as the remnant of an older outflow in the bottom right of the first two panels. For reference, the point of coalescence for model co-BH is $\mathrm{T}=1.5 \mathrm{Gyr}$.

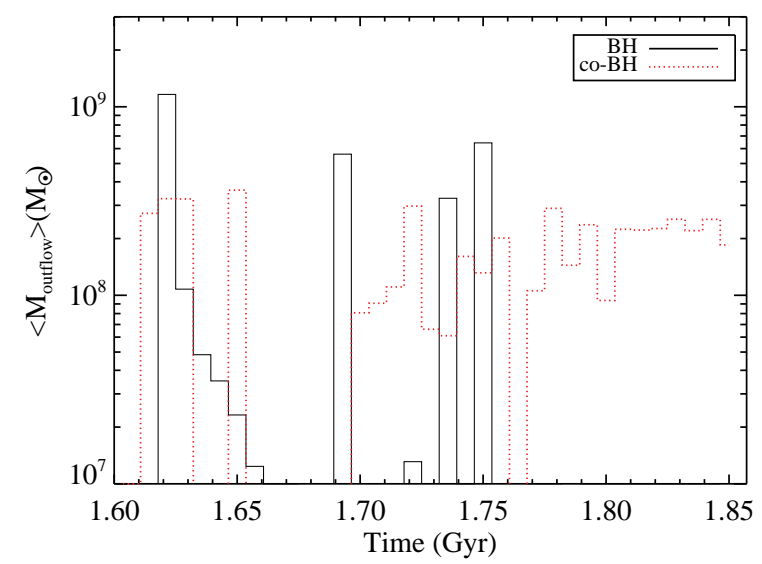

FIG. 15.- Masses of detectable outflows as a function of time for model $\mathrm{BH}$ and co-BH (thus varying only merger orbit angle) averaged over three orthogonal sightlines The masses of a typical outflow are comparable between models, though the coplanar model drives outflows for longer periods of time.

strongly enough to be seen in the emission spectrum over the main Gaussian. In the remainder of $\S 5$, when we discuss clumps of outflowing and infalling gas, we will be referring to those above this minimum mass that are able to impact the emission line profile.

Because high velocity peaks appear in our simulations even in spatially unresolved observations, they may be a powerful diagnostic for AGN and starburst induced outflows both in the local Universe and at earlier epochs. However, at this point in the discussion, there are open questions regarding the uniqueness of this line profile, as well as how these secondary peaks specifically relate to starburst and AGN activity. In the remainder of this section, we attempt to quantify the use of molecular emission line profiles as a diagnostic for outflowing gas in galaxy mergers.

\subsection{The Origin of High Velocity Peaks in CO Emission Profiles}

Emission lines representing gas with a strong line of sight velocity component can originate from either outflowing or infalling gas. As demonstrated by Narayanan et al. (2006a), AGN feedback-driven molecular outflows can generate high
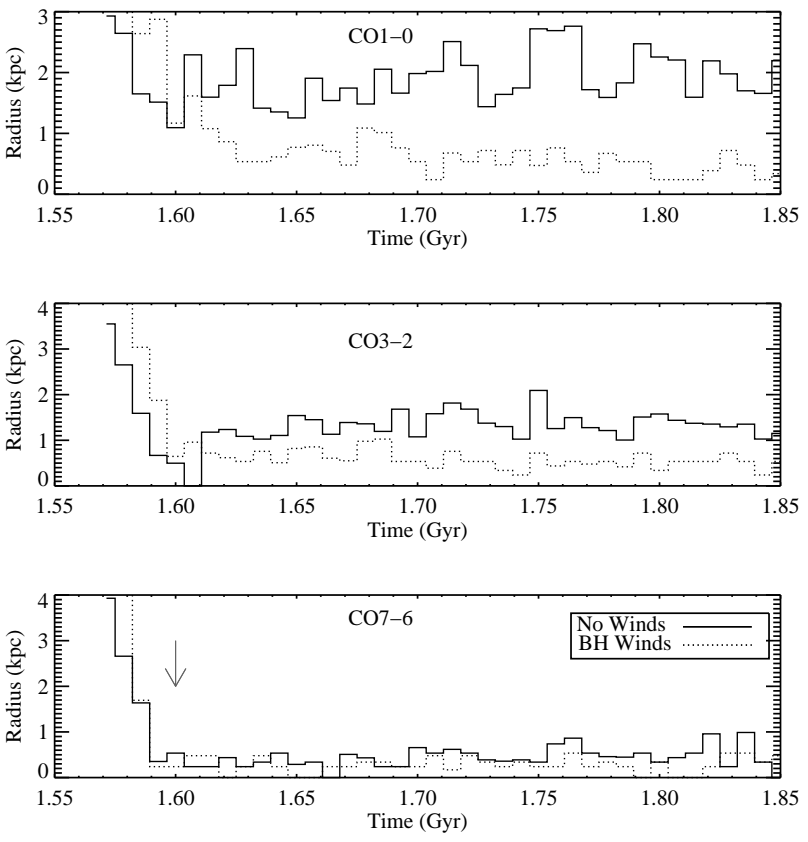

FIG. 16. - CO halflight radius as a function of time for models with AGN winds only (model $\mathrm{BH}$ ) and with no winds (model no-winds) for observable $\mathrm{CO}$ transitions $\mathrm{CO}(\mathrm{J}=1-0), \mathrm{CO}(\mathrm{J}=3-2)$ and $\mathrm{CO}(\mathrm{J}=7-6)$. The emission from model $\mathrm{BH}$ with winds is uniformly compact in all $\mathrm{CO}$ transitions, typically confined to a region of a few hundred pc. The emission from model no-winds, without winds, is stratified, with a characteristic $\mathrm{CO}(\mathrm{J}=1-0)$ scale of $\sim 1.5-2$ $\mathrm{kpc}$, and becoming more compact at higher lying transitions. The arrow in the third panel denotes the point of nuclear coalescence. (See text for details.)

Any gas falling into the central potential with a negative radial velocity with respect to the center of mass of the system (e.g gas that is in infall, or pre-coalescence mergers) may also drive high velocity peaks in the $\mathrm{CO}$ emission lines, and are thus degenerate with high velocity peaks that originate in outflows. It is therefore necessary to establish the uniqueness of high velocity peaks in $\mathrm{CO}$ emission lines with respect to an actual outflow or infall origin. Any attempt to break this degeneracy requires a more detailed knowledge of the physical properties and observational characteristics of the galaxy during periods when high velocity peaks are observable.

In order to assess how the evolutionary state of the galaxy merger may determine the origin of high velocity peaks (i.e. 


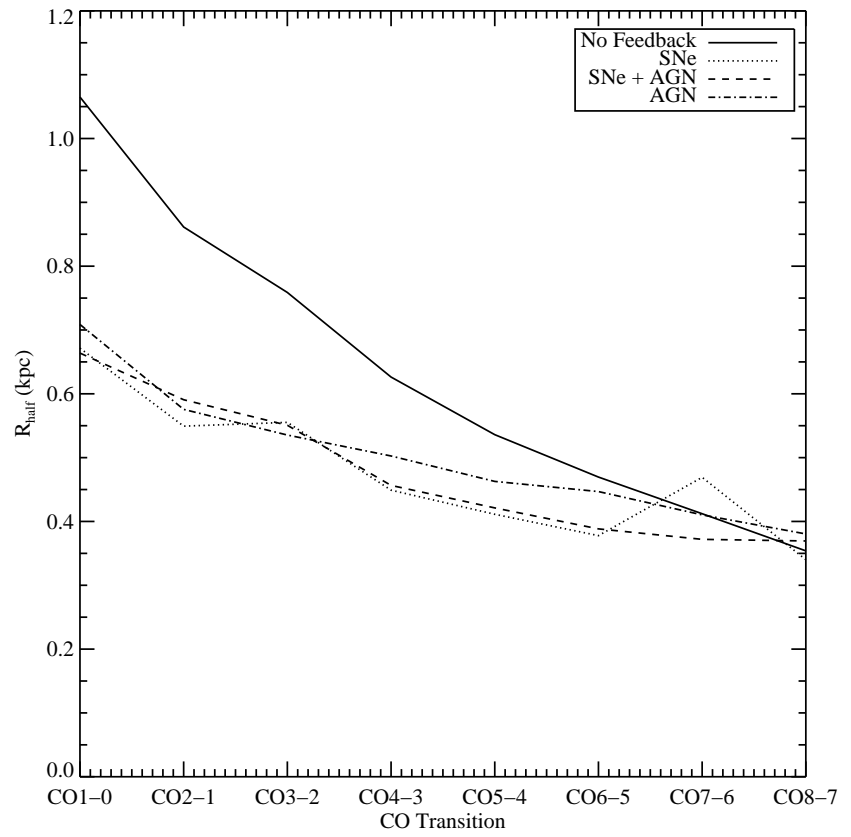

FIG. 17.- Average halflight radius as a function of transition for models $\mathrm{BH}$, no-winds, sb and sbBH (thus varying only wind parameters). The average halflight radius is calculated after nuclear coalescence. The emission from the model without winds is relatively stratified, with emission becoming more compact at higher transitions. The models with winds also exhibit stratification, though the halflight radius for the lowest transitions is about a factor of two smaller than the models without winds.

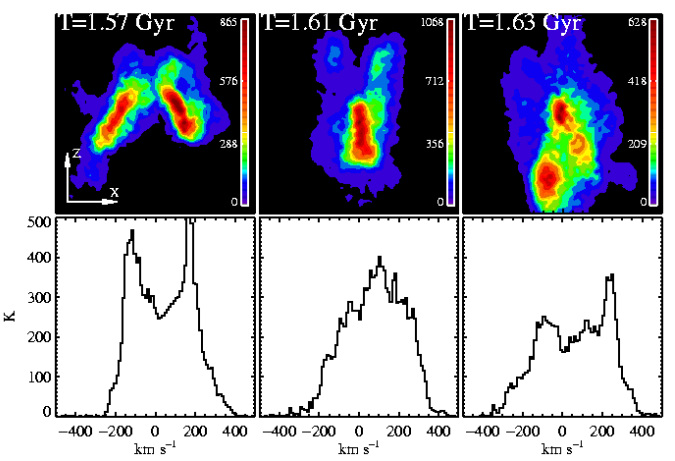

FIG. 18. - top: $\mathrm{CO}(\mathrm{J}=1-0)$ emission contours of three snapshots during the merger simulation with winds from black holes (model $\mathrm{BH})$. bottom: $\mathrm{CO}$ $(\mathrm{J}=1-0)$ spectra of snapshots, viewing the emission from the $-\hat{z}$ direction (coordinate axes in top left panel). The $\mathrm{CO}$ emission lines early in the merger are characteristically double-peaked, where each peak corresponds to emission from the individual nuclear regions of the progenitor galaxies (first column). As the merger advances, the emission lines are typically well-described by a single Gaussian (middle column). There are excursions from this trend, however, as outflowing gas can give rise to a secondary peak in the emission line red or blueshifted from the systemic velocity of the galaxy (third column; this outflow and line profile was also presented in Narayanan et al. 2006a). The emission contours each have their own scale on the right, to facilitate color contrasts. For reference, the time of nuclear coalescence is $\mathrm{T}=1.6 \mathrm{Gyr}$. The units of emission are in velocity-integrated intensity $\left(\mathrm{K}-\mathrm{km} \mathrm{s}^{-1}\right)$. The panels are $12 \mathrm{kpc}$ on a side.

exhibits high velocity peaks. To this end, we created synthetic $\mathrm{CO}(\mathrm{J}=1-0)$ spectra along three orthogonal sightlines for the models in Table 1. These spectra were made by integrating over the entire $\mathrm{CO}$ emitting area. To identify candidate emission lines with high velocity peaks driven by winds

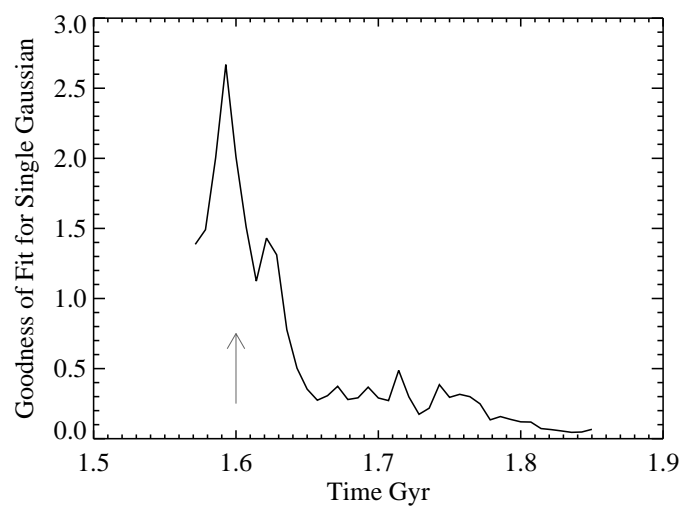

FIG. 19.- Goodness of fit for a single Gaussian fit to spectra from model $\mathrm{BH}$ as a function of time. When the merger is prior to and near coalescence, and the gas is highly dynamical, and a single Gaussian provide a relatively poor description of the emission line profile. As the gas virializes after coalescence, a single Gaussian better represents the emission line profile. The goodness of fit is taken here as the median of the square of the residuals. The arrow denotes the time of nuclear coalescence.

ditional peak with velocity greater than $\pm 160 \mathrm{~km} \mathrm{~s}^{-1}$. This fiducial requirement is set in place to filter out multiple emission peaks that arise from rotation as $\sim 160 \mathrm{~km} \mathrm{~s}^{-1}$ is the circular velocity of the post-merger galaxy at the spatial extent of the $\mathrm{H}_{2}$ gas, and thus the maximum velocity of rotating gas. We consider all high velocity peaks whose peak intensity is at least $20 \%$ the peak line intensity.

Upon identifying a line with a high velocity peak, we then determine whether the clump of gas generating the high velocity peak is outflowing or infalling. In order to simplify the analysis, in the few cases with multiple high velocity peaks, we focused on the peak with highest velocity. In Figure 20 , we plot a histogram of the number of high velocity peaks seen as a function of time in the simulation with black hole winds only (model BH), summed over 3 orthogonal lines of sight. We have further broken the line profiles down into having an outflow or infall origin. As a reference for the evolutionary state of the merger, we have overplotted the SFR, black hole accretion rate, and bolometric luminosity.

Figure 20 demonstrates that while the number of high velocity peaks is roughly constant after the major merger, the origin of these line profiles is a function of the evolutionary state of the galaxy. During phases of heavy black hole growth, the majority of the high velocity peaks owe to outflowing gas. Towards the end of the quasar phase, when much of the blowout has already occurred, gas raining from outer parts of the galaxy give rise to multi-peaked profiles with an infall origin. Conversely, similar analysis from the model without winds shows a more random origin for high velocity peaks in the emission profiles, reflecting the more randomly oriented velocity vectors of loosely bound clumps. This is explicitly shown in Figure 21, where we show the origin of high velocity peaks in a merger without winds compared with the bolometric and IR luminosity.

Another way of saying this is that the winds in model $\mathrm{BH}$ do not cause the high velocity peaks to exist in the $\mathrm{CO}$ spectra. Rather winds typically entrain the clumps of gas behind the high velocity peaks in outflow, thus giving physical significance to secondary peaks. This is not the case in the model with no winds, where the molecular gas representative of the 
models both with and without winds; in wind models, though, these peaks preferentially owe to outflowing gas during peaks of starburst activity and black hole growth.

Observationally, many of the criteria that are used in creating samples of local galaxy mergers may select galaxies which are at the peak of their starburst or AGN activity. High velocity peaks in the emission profiles (e.g. higher than the circular velocity of the host) observed in these galaxies may be likely, then, to owe to outflowing clumps of gas (Figure 20.). For example, the extreme bolometric and IR luminosities trace not only the prodigious star formation owing to the merger induced starburst, but also reflect a large contribution from the growing supermassive black hole. The winds associated with this black hole growth are behind much of the outflowing molecular gas during the most luminous phase of the galaxy's evolution. Similarly, IR colors which select objects with embedded AGN, such as the $25 \mu \mathrm{m} / 60 \mu \mathrm{m}$ flux ratio, may preferentially show objects which owe their outflow profile to outflows as opposed to infalling gas clumps. Finally, the hard X-ray flux is dominated by the central AGN, and thus traces the general growth of the central black hole. Our models suggest that the phase during which the X-ray luminosity is at its greatest will be marked by high velocity peaks in the $\mathrm{CO}$ emission originating in outflowing molecular gas.

\subsection{Distinguishing Characteristics}

A natural question is whether or not one can observationally infer the nature of high velocity peaks in $\mathrm{CO}$ emission lines as discussed in $\$ 5.1$ Specifically, high velocity peaks can originate in both wind-driven outflows, as well as owing to dynamical effects in merging galaxies (e.g. Figures 20 and 21). Distinguishing characteristics between the various origins for high velocity peaks may be evident when considering limiting velocities for the high velocity peak much greater than the virial velocity. Figures 20 and 21 showed the presence of all high velocity peaks beyond a limiting velocity being set at the virial velocity of the galaxy (here, $\sim 160 \mathrm{~km} \mathrm{~s}^{-1}$ ). When considering the origin of high velocity peaks driven by dynamical effects, few emitting clumps of gas are able to regularly sustain velocities much greater than the escape velocity of the galaxy (or $\sqrt{2} \times V_{c}$ ). In contrast, when included, winds are able to drive outflows at higher speeds.

We show this effect quantitatively in Figure 22 where we plot the number of high velocity peaks as a function of limiting velocity for both two models with AGN winds (models $\mathrm{BH}$ and co-BH) and the model with no winds. At velocities greater than $\sim 1.7$ times the virial velocity, the number of high velocity peaks driven by purely dynamical effects in model no-winds drops rapidly while winds from the AGN in model $\mathrm{BH}$ and co-BH are still able to drive clumps in outflow at these velocities. The range of the numbers of high velocity peaks in the models with AGN winds owes to a merger-orientation dependent range in instantaneous black hole feedback energies. More efficient fueling of the central AGN in the coplanar model allows for a stronger impact of the winds on the molecular line profiles (cf. \$4.2.3). In either case, both models which consider AGN feedback show that AGN feedbackdriven winds can drive high velocity peaks at larger velocities than dynamical effects can typically account for. In this sense, one may be able to discriminate between high velocity peaks with an outflow origin versus random kinematics when restricting the limiting velocity of the peaks to $\gtrsim 1.7$ times the ined in this work, and may not necessarily be general.

The dispersion seen between the no winds model and the models with AGN winds ( $\mathrm{BH}$ and co-BH) in Figure 22 is in principle a lower limit. The galaxies in Figure 22 are relatively low mass $-M_{\star} \sim 10^{11} \mathrm{M}_{\odot}$. Cox et al. (2007) found that the energy input by accreting black holes in galaxy mergers increases as a function of galaxy mass for a variety of progenitor gas fractions (ranging from 0.05 to 0.8 ) and initial merger angles. Specifically, these authors found $E_{\mathrm{BH}} / f_{g} \propto M_{\star}^{1.13}$, suggesting that higher mass galaxies have the capacity to drive CO peaks at even higher speeds compared to their virial velocity. As an example, a merger with similar gas fraction and orientation angle as model $\mathrm{BH}$, though with final stellar mass $\sim 10^{12}$ would have roughly an order of magnitude greater black hole energy input (Cox et al. 2007).

Figure 22 suggests that the effect of AGN feedback-driven winds on $\mathrm{CO}$ emission line profiles should be discernible by comparing a merger's virial velocity to observed high velocity features in the CO line profile. A comparably clear (and observationally more tractable) test may be an analysis of the velocity offset of high velocity peaks with respect to the centroid velocity of the emission line. When examining high velocity peaks defined with respect to the centroid velocity, we find the curves presented in Figure 22 are robust. This owes to a narrow distribution of centroid velocities in all the models, with a typical standard deviation in the distribution $\sigma \sim 20 \mathrm{~km} \mathrm{~s}^{-1}$.

\section{DISCUSSION}

Many of the model results presented here have the potential to be directly tested in the near future, either with existing datasets, or with observations from millimeter-wave interferometers such as CARMA and PdBI, and high resolution submillimeter telescopes such as the Submillimeter Array (SMA) or Atacama Large Millimeter Array (ALMA).

First, it is worth briefly revisiting the evolutionary status of the galaxies studied here, and placing them into a larger context. In a merger-driven scenario for quasar formation, gaseous inflows fueled by tidal torquing on the gas (Barnes \& Hernquist 1991, 1996; Hernquist, 1989) fuel nuclear starbursts (Mihos \& Hernquist, 1996), allowing the galaxy to be viewed as a dusty starburst system similar to local ULIRGs (Chakrabarti et al. 2007a). The gaseous inflows fuel central black hole growth (Hopkins et al. 2005a-d; 2006a-d; 2007h; Di Matteo et al. 2005; Springel et al. 2005a) which makes the galaxy first visible as a ULIRG with warm infrared colors (Chakrabarti et al. 2007a), and then as an optically selected quasar (Hopkins et al. 2005a-d; 2006a-d; 2007h). A key physical process in driving the host galaxy along this sequence is galactic winds. Namely, winds driven by AGN feedback (as well as nuclear starbursts) contribute to clearing the veil of obscuring gas and dust surrounding the central black hole, and allow the quasar to become visible across numerous sightlines (Hopkins et al. 2005a). These winds are most prominent during the $\sim 200 \mathrm{Myr}$ associated with major black hole growth. The models presented in this work therefore examine simulated galaxy mergers during this time period of heightened wind activity, starting near nuclear coalescence, and ending slightly after the quasar light curve has declined. During this time, the galaxy is seen to have increased IR, Xray and $B$-band luminosities, indicative of the starburst event and central black hole growth (Figure 20). In this sense, these simulations are designed to provide analysis for local galaxies 

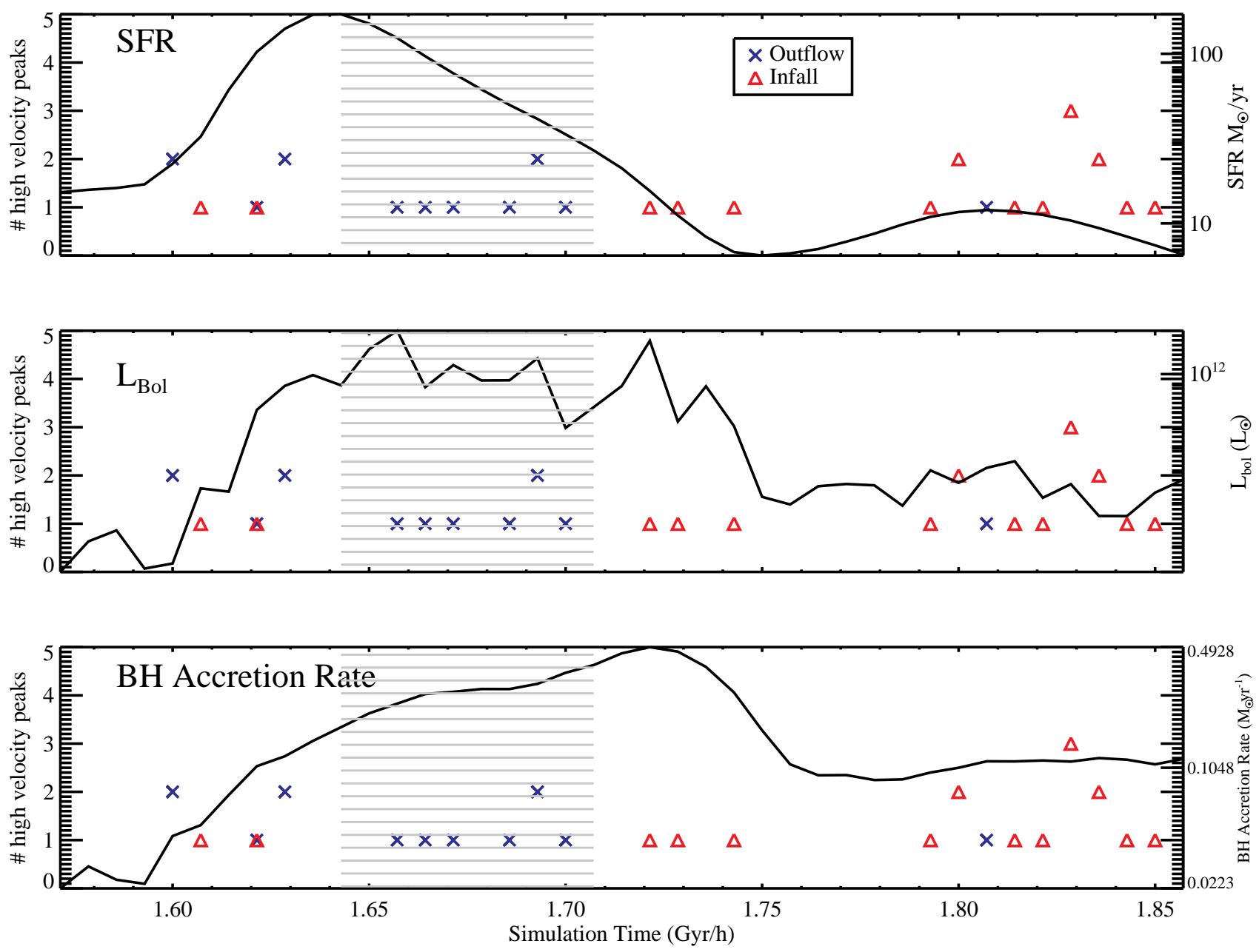

FIG. 20.- Physical and observable properties of model BH (model with BH winds) as a function of simulation time. Additionally plotted are a histogram of the number of secondary high velocity peaks seen, and the physical origin of the peak. The blue crosses are number of high velocity peaks associated with an outflow (summed over 3 orthogonal viewing angles), and the red triangles are number of high velocity peaks originating from infalling gas. Infalling gas is seen both early and late in the merger's evolution. During periods of elevated activity (e.g. increased SFR or black hole accretion), most of the high velocity peaks owe to outflowing gas (hatched region). The left axis is associated with the histogram of high velocity peaks, and the right axis with the physical or observable quantity overplotted in that panel. For reference, the time of coalescence is $\mathrm{T}=1.6 \mathrm{Gyr}$.

Second, we have shown that the effects of winds can shape the molecular morphology of galaxy mergers. For example, distinct differences are seen in the simulations with winds versus without when considering the spatial extent of the $\mathrm{CO}$ emitting gas ( $\$ 4.3$ ). The predicted weak evolution of average half-light radius as a function of transition for evolved mergers can be tested in the $\mathrm{CO}(\mathrm{J}=1-0),(\mathrm{J}=2-1),(\mathrm{J}=3-2)$ and $(\mathrm{J}=7-$ 6) transitions with current technology using a millimeterwave interferometer (e.g. CARMA, PdBI) and the SMA.

Perhaps the most dramatic effects of galactic winds on the $\mathrm{CO}$ morphology lie in signatures of entrained molecular outflows in emission contour maps. $\mathrm{CO}$ outflows driven by starburst-dominated winds have been imaged in a handful of nearby galaxies. Recent mapping of starburst galaxies M82 and NGC 3256 have shown that molecular gas can indeed survive in outflows entrained in starburst-driven winds (Walter et al, 2002; Sakamoto et al. 2006). Both galaxies are known to be interacting systems, the latter a major merger. The outflow masses are recorded to be $\sim 10^{7}-10^{8} \mathrm{M}_{\odot}$, consistent with the tected in $\mathrm{CO}(\mathrm{J}=2-1)$ emission (peaking at $\mathrm{CO} \mathrm{J} \sim 3$ or 4 ; Weiß et al. 2005b). The outflow observed in M82 appears to be distributed in a roughly spherical halo with radius $\sim 1.5 \mathrm{kpc}$. This outflow may correspond with an $\mathrm{H} \alpha$ outflow, as well as M82's dust halo (Alton, Davies \& Bianchi, 1999; Walter et al. 2002). This type of morphology is seen along some sightlines in the models $\mathrm{BH}$ and co- $\mathrm{BH}$, alongside the more collimated outflows characteristic of e.g. Figure 12

Outflows which may be driven by a central AGN have been imaged in local galaxies as well. Recent SMA observations of NGC 6240 by Iono et al. (2007) have revealed an outflow in $\mathrm{CO}(\mathrm{J}=3-2)$ morphologically similar to the outflow seen in Figure 10. NGC 6240 is a major merger near final coalescence with two central X-ray sources, which Chandra observations have suggested are a binary AGN (Komossa et al, 2003). In this sense, the outflow imaged in NGC 6240 occurs when our models suggest the most massive outflows may be imageable $(\$$ 4.2.2). The mass of the outflow in this system is estimated to be $\sim 5 \times 10^{8} \mathrm{M}_{\odot}$, consistent with the typical masses 

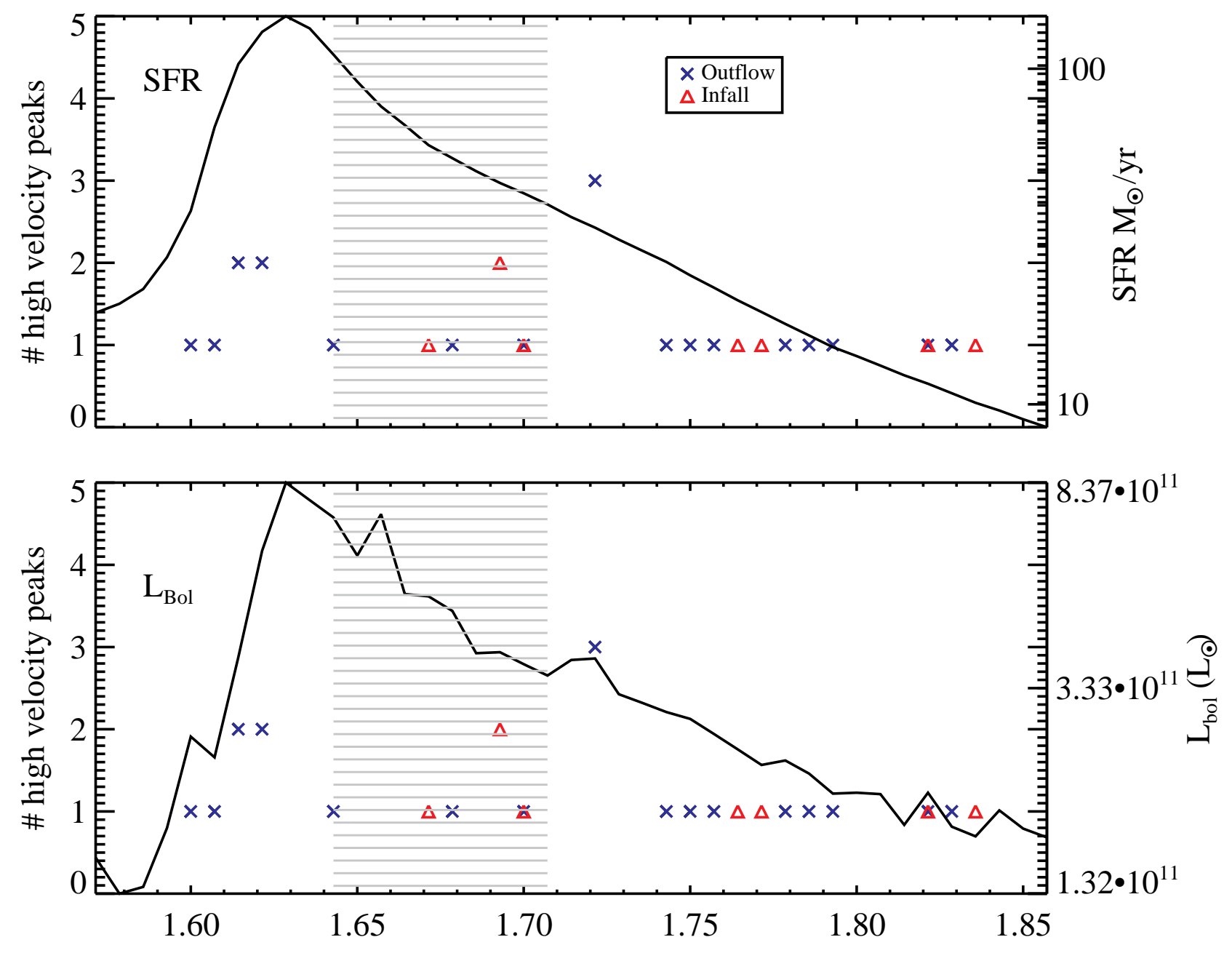

FIG. 21. - SFR and bolometric luminosity from model no-winds as a function of simulation time. Additionally plotted are a histogram of the number of secondary high velocity peaks seen, and the physical origin of the peak. The blue crosses are number of high velocity peaks associated with an outflow (summed over 3 orthogonal viewing angles), and the red triangles are number of high velocity peaks originating from infalling gas. No apparent trends are seen reflecting the randomly oriented radial velocity vectors driving the high velocity peaks in the no-winds model. The hatched region covers the same time period as that in Figure 20 just as a comparative reference with that plot. The left axis is associated with the histogram of high velocity peaks, and the right axis with the physical or observable quantity overplotted in that panel. For reference, the time of coalescence is $\mathrm{T}=1.6 \mathrm{Gyr}$.

logical features to those associated with molecular outflows have been detected in local LIRG/ULIRG NGC 985, though the interpretation of these features is still uncertain (Appleton et al. 2002).

While the number of outflows directly imaged in $\mathrm{CO}$ remains small and limited to only relatively recent observations, outflows traced in absorption (e.g. Na I D lines) have a much more extensive detection history and associated literature. Absorption line outflows have been detected in the UV and optical in both nearby starburst galaxies/AGN (Heckman et al. 2000; Martin, 2005; Rupke \& Veilleux, 2005; Rupke, Veilleux \& Sanders, 2005a-c), as well as high-redshift galaxies (Pettini et al. 2002; Shapley et al. 2003) and quasars (Ganguly et al. 2006; Hamann, Barlow \& Junkkarinen, 1997; Misawa et al. 2005, 2007a,b; Narayanan et al. 2004; Trump et al. 2006; Wise et al. 2004). HI has also been seen in outflow in active galaxies via absorption lines (Morganti, Tadhunter $\&$ Oosterloo; 2005). In addition to the neutral absorption line outflows detected, many outflows are relatively high ioniza- al. 2004). In this sense, it appears as though these may be different populations of outflows from the cold, neutral emission line outflows discussed in this work. Interestingly, in the few systems with $\mathrm{CO}$ outflow candidates (as evidenced by their morphology), there is an apparent coincidence between the velocity offsets in the molecular outflows and absorption line outflows. For example, the starburst-driven outflow observed in local merger NGC 3256 by Sakamoto et al. (2006) has a velocity offset (from the systemic, which in the case of $\mathrm{CO}$, typically corresponds to the nuclear emission) of $\sim 300 \mathrm{~km} / \mathrm{s}$, while Heckman et al. (2000) showed a blueshifted Na I D absorption line of velocity $\sim 300 \mathrm{~km} / \mathrm{s}$. Similarly, Appleton et al. (2002) found two Ly $\alpha$ and NV absorbers in NGC 985 at coincident velocities as those which $\mathrm{CO}$ gas is seen projected against the nucleus. The data bring up the interesting speculative suggestion that perhaps the absorption line outflows seen in these systems are physically associated with the $\mathrm{CO}$ emitting outflows. If so, it may be that the neutral and ionized outflows exist more toward the outer envelopes of the entrained 


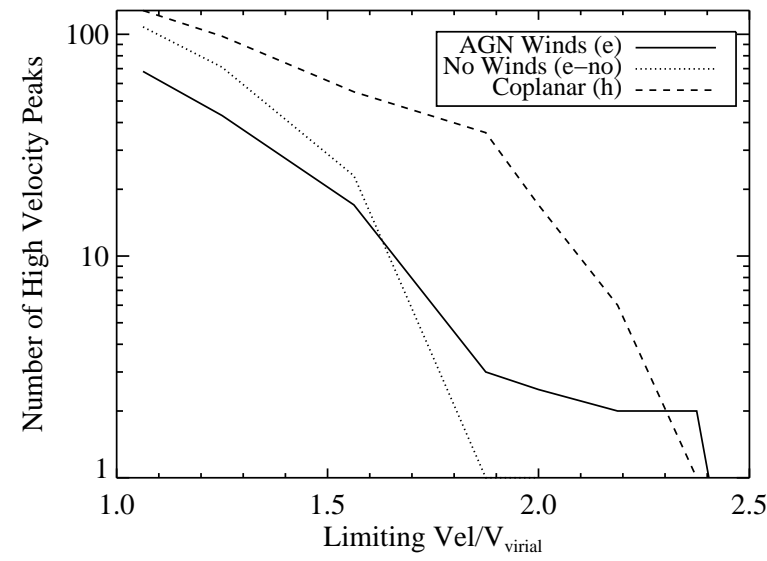

FIG. 22.- Number of high velocity peaks as a function of minimum velocity for the high velocity peak summed over three orthogonal sightlines. Solid line is model with AGN winds (model $\mathrm{BH}$ ) and dashed line is model with no winds. High velocity peaks in model without winds are driven by random dynamics of clumps of gas, and thus are unable to sustain velocities much greater than the virial velocity of the host galaxy. In contrast, clumps of gas entrained in AGN winds are seen at higher velocities, giving rise to more high velocity peaks at the highest velocities. One may be able to discriminate between high velocity peaks with an outflow origin versus random kinematics when restricting the limiting velocity $\gtrsim 1.7$ times the virial velocity.

central regions of these flows are extremely cold and dense ( $\sim 10-30 \mathrm{~K}, \sim 10^{5} \mathrm{~cm}^{-3}$; Figure 10 ). A full investigation into the potential relationship between UV and optical absorbers and $\mathrm{CO}$ outflows is beyond the scope of this work, but studies into possible physical motivations for absorption line outflows within the context of these models are underway.

In principle, unresolved emission line profiles from galaxies are more easily obtainable in large numbers than the highresolution maps necessary to directly image outflows. Brief inspection by eye suggests that at least some local galaxies appear to espouse the high velocity peaks discussed in $\S 5$ (e.g. Narayanan et al. 2005; Sanders Scoville \& Soifer, 1991; Solomon, Downes \& Radford, 1992; Yao et al. 2003). As previously discussed, robustly determining the physical mechanism driving high velocity peaks is difficult, although tests similar to Figure 22 may be possible with some prior knowledge of the host circular velocity. It is interesting to note that absorption line outflows, which have been attributed to starburst-driven winds, have been detected in many of the ULIRGs in the aforementioned CO samples (Martin, 2005; Rupke et al. 2005a-c).

Apparent high velocity peaks appear prodigiously in $z \sim 2$ submillimeter Galaxies (SMGs; Greve et al. 2005; Tacconi et al. 2006; see Blain et al., 2002 and Solomon \& Vanden Bout 2006 for recent reviews). If the high velocity peaks in SMGs owe to outflows, they may be explained by the prodigious star formation rates in these galaxies, in combination with a potential contribution from an embedded AGN. For example, if the extreme luminosities owe solely to starbursts, then massive star formation rates of order $\sim 1000 \mathrm{M}_{\odot} \mathrm{yr}^{-1}$ are typical of these sources (e.g. Blain et al. 1999). The relative role of AGN energy input in SMGs is a debated topic, as well as whether they are currently undergoing, or are soon to undergo a heavy black hole accretion phase (e.g. Alexander et al. 2005a,b; Borys et al, 2005; Chakrabarti et al. $2007 b)$. In either case, powerful winds produced by the in- tion against a direct application of these model results toward SMGs. For one thing, the virial properties of the progenitor galaxies in these models have not been explicitly scaled for $z \sim 2$ (Robertson et al. 2006a), and it is not clear how that will affect merger-induced star formation rates and black hole growth during the active period. Moreover, it is not completely clear that SMGs are the product of binary mergers as this work has focused on, and the kinematic profiles in these sources may be rather complex. Models appropriate for $z \sim 2$ mergers (e.g. Chakrabarti et al. 2007b; Robertson et al. 2006a) will have to be studied in the context of galactic winds in order to asses the potential relationship between these results and SMGs.

\section{CONCLUSIONS AND SUMMARY}

We have combined 3D non-LTE radiative transfer calculations with SPH simulations of galaxy mergers in order to investigate the effects of galactic-scale winds on the molecular line emission in starburst galaxies and AGN. We find that galactic winds are a natural result of merger-induced star formation and black hole growth. These winds can entrain molecular gas of order $\sim 10^{8}-10^{9} \mathrm{M}_{\odot}$ which imprints generic signatures in both the $\mathrm{CO}$ morphology as well as unresolved emission line profiles. The specifics of the morphological and emission line indicators of molecular outflows depend on physical parameter choices within the galaxy merger models. In particular, the energy source (i.e. BH accretion or star formation), as well as the merger orientation can vary the strength, direction, and duration of molecular outflows in emission contour maps, as well as the velocity separation of high velocity peaks in multi-component emission lines. Many of the results including the halflight radius as a function of excitation, or the velocity offsets of high velocity peaks in the emission lines vary enough between physical parameter choices such that these models may be used to constrain physical origins for observed $\mathrm{CO}$ morphologies and line profiles. In detail, we find the following:

1. Molecular outflows entrained in AGN feedback and starburst-driven winds can be directly imageable via $\mathrm{CO}$ emission line mapping. These types of outflows have been recorded in at least some local systems (e.g. Iono et al. 2007). These outflows will be detectable at cosmological distances given the predicted spatial resolution of ALMA.

2. Molecular outflows entrained in winds driven by AGN feedback are typically longer lived than those entrained in starburst driven winds. This owes to the relative strength of AGN feedback-driven winds versus starburst driven winds.

3. Winds from AGN feedback in coplanar merger models are typically more powerful than mergers which occur at more random orientations. This results in outflows in these models being visible for the majority of the $\sim 200$ Myr 'active period' studied here.

4. The spatial extent of CO emission can be controlled by the presence of galactic winds. The emission is seen to be stratified with transition in all models such that the $\mathrm{CO}(\mathrm{J}=1-0)$ halflight radius is larger than the radius from higher lying transitions (e.g. CO J=7-6), though 
emission in local mergers $\left(\mathrm{R}_{1 / 2}\right.$ typically confined to the central kpc; Scoville \& Bryant, 1999) may be a consequence of AGN feedback or starburst-driven winds.

5. In all models, high velocity peaks (peaks at velocities greater than the circular velocity) can exist superposed on the post-merger galaxy's broad CO emission line. In models without winds, these peaks owe to random kinematics of molecular gas. In models with winds, these peaks are seen to originate primarily from gas entrained in outflow, at least during the period of peak black hole accretion/star formation.

6. For the models studied here (Table 1), high velocity peaks driven by random kinematics do not typically appear at velocity offsets (from systemic) greater than $\sim 1.7$ times the circular velocity of the post-merger galaxy. In contrast, peaks entrained in AGN feedbackpowered winds can be driven to velocities near 2.5 times the circular velocity. The centroid velocities of the simulated lines are typically $(1 \sigma)$ within $\sim 20$ $\mathrm{km} \mathrm{s}^{-1}$ of the true systemic velocity, and can generally be used as a reliable substitute for the systemic velocity in these models. Thus the above results hold true when measuring the velocity offsets of high velocity peaks with respect to line centroids as well.

We thank John Bieging, Sukanya Chakrabarti, Arjun Dey, Chris Groppi, Fred Hamann, Daisuke Iono, Casey Papovich, Yancy Shirley, and Linda Tacconi for helpful conversations. DN was funded for this work by an NSF Graduate Research Fellowship. The calculations were performed at the HarvardSmithsonian Center for Parallel Astrophysical Computing. BR was supported in part by NASA through the Spitzer Space Telescope Fellowship Program. Support for this work was also provided by NASA through grant number HST-AR10308 from the Space Telescope Science Institute, which is operated by AURA, Inc. under NASA contract NAS5-26555.

\section{REFERENCES}

Alexander, D.M., Bauer, F.E., Chapman, S.C., Smail, I., Blain, A.W., Brandt, W.N., Ivison, R.J., 2005a, ApJ, 632, 736

Alexander, D.M., Smail, I., Bauer, F.E., Chapman, S.C., Blain, A.W., Brandt, W.N., Ivison, R.J., 2005b, Nature, 434, 38

Alton, P.B., Davies, J.I., Bianchi, S., 1999, A\&A, 343, 51

Andre, P., Ward-Thompson, D., Motte, F., 1996, A\&A, 314, 625

Appleton, P., Charmandaris, V., Gao, Y., Combes, F., Ghigo, F., Horellou, C., Mirabel, I.F., 2002, ApJ, 566, 682

Armus, L., et al. 2004, ApJS, 154, 178

Armus, L., Bernard-Salas, J., Spoon, H.W.W., Marshall, J.A., Charmandaris, V., Higdon, S.J.U., Desai, V., Hao, L., Teplitz, H.I., Devost, D., Brandl, B.R., Soifer, B.T., Houck, J.R., 2006, ApJ, 640, 204

Barnes, J.E., Hernquist, L.E., 1991, ApJ, 370, L65

Barnes, J.E., Hernquist, L.E., 1996, ApJ, 471, 115

Bayet, E., Gerin, M., Phillips, T.G., \& Contursi, A., 2004, A\&A, 427, 45

Bernes, C., 1979, A\&A, 73, 67 Scoville, N.Z., 1999, AJ, 117, 2632

Blain, A.W., Kneib, J.-P., Ivison, R.J., Smail, I., 1999, ApJ, 512, L87

Blain, A.W., Smail, I., Ivison, R.J., Kneib, J.-P., Frayer, D.T., 2002, PhR, 369 , 111

Blitz, L., Fukui, Y., Kawamura, A., Leroy, A., Mizuno, N., Rosolowsky, E., 2006, "Giant Molecular Clouds in Local Group Galaxies", in Protostars and Planets $V$, [astro-ph/0602600]

Bondi, H., Hoyle, F., 1944, MNRAS, 104, 273

Borys, C., Smail, I., Chapman, S.C., Blain, A.W., Alexander, D.M., Ivison, R.J., 2005, ApJ, 635, 853

Bradford, C.M., Nikola, T., Stacey, G., Bolatto, A.D., Jackson, J.M., Savage, M.L., Davidson, J.A., Higdon, S.J., 2003, ApJ, 586, 891

Bradford, C.M., Stacey, G.J., Nikola, T., Bolatto, A.D., Jackson, J.M., Savage, M.L., Davidson, J.A., 2005, ApJ, 623, 866

Bryant, P., Scoville, N., 1999, AJ, 117, 2632

Chakrabarti, S., Cox, T.J., Hernquist, L., Hopkins, P.F., Robertson, B., Di Matteo, T., 2007a, ApJ, 658, 840

Chakrabarti, S., Fenner, Y., Hernquist, L., Cox, T.J., Hopkins, P.F., 2007b, ApJ, submitted astro-ph/0610860

Combes, F., Maoli, R., Omont, A., 1999, A\&A, 345, 369

Cox, T.J., Jonsson, P., Primack, J.R., Somerville, R.S., 2004, ApJ, 607, L87

Cox, T.J., Dutta, S.N., Di Matteo, T., Hernquist, L., Hopkins, P.F., Robertson, B., Springel, V., 2006a, ApJ, 650, 791

Cox, T.J., Di Matteo, T., Hernquist, L., Hopkins, P.F., Robertson, B., Springel, V., 2006b, ApJ, 643, 692

Cox, T.J., Jonsson, P., Primack, J.R., Somerville, R.S., 2006c, MNRAS, 373, 1013

Cox, T.J., Chakrabarti, S., Di Matteo, T., Hernquist, L., Hopkins, P.F., Krause, E., Li, Y., Robertson, B., Springel, V., 2007, ApJ, submitted

de Grijp, M.H.K., Miley, G.K., Lub, J., de Jong, T., 1985, Nature, 314, 240

Di Matteo, T., Springel, V., Hernquist, L., 2005, Nature, 433, 604

Downes, D., Solomon, P.M., 1998, ApJ, 507, 614

Elmegreen, B., Falgarone, E., 1996, ApJ, 471, 816

Fabian, A.C., 1999, MNRAS, 308, L39

Farrah, D., Afonso, J., Efstathiou, A., Rowan-Robinson, M., Fox, M.,
Fuller, G.A., Myers, P.C., 1992, ApJ, 384, 523

Ganguly, R., Sembach, K.R., Tripp, T.M., Savage, B.D., Wakker, B.P., 2006, ApJ, 645, 868

Gao, Y., Solomon, P.M., 1999, ApJ, 512, L99

Gebhardt, K., Bender, R., Bower, G., Dressler, A., Faber, S.M., Filippenko, A.V., Green, R., Grillmair, C., Ho, L.C., Kormendy, J., Lauer, T.R., Magorrian, J., Pinkney, J., Richstone, D., Tremaine, S., 2000, 539, L13

Greve, T., Bertoldi, F., Smail, I., Neri, R., Chapman, S.C., Blain, A.W., Ivison, R.J., Genzel, R., Omont, A., Cox, P., Tacconi, L., Kneib, J.-P., 2005, MNRAS, 359, 1165

Greve, T., Sommer-Larsen, J., 2006, ApJ, in press astro-ph/0608683

Hamann, F., Barlow, T.A., Junkkarinen, V., 1997, ApJ, 478, 87

Heckman, T.M., Lehnert, M.D., Strickland, D.K., \& Armus, L., 2000, ApJS, 129,493

Hernquist, L., 1989, Nature, 340, 687

Hernquist, L., 1990, ApJ, 356, 359

Hernquist, L., Quinn, P.J., 1987, ApJ, 312, 1

Hernquist, L., Spergel, D.N., 1992, ApJ, 399, L117

Hollenbach, D.J., Werner, M.W., \& Salpeter, E.E., 1971, ApJ, 163, 165

Hollenbach, D. J., \& Tielens, A. G. G. M. 1999, Reviews of Modern Physics, 71,173

Hopkins, P.F., Hernquist, L., Martini, P., Cox, T.J., Robertson, B., Di Matteo, T., Springel, V., 2005a, ApJ, 625, L71

Hopkins, P.F., Hernquist, L., Cox, T.J., Di Matteo, T., Martini, P., Robertson, B., Springel, V., 2005b, ApJ, 630, 705

Hopkins, P.F., Hernquist, L., Cox, T.J., Di Matteo, T., Robertson, B., Springel, V., 2005c, ApJ, 630, 716

Hopkins, P.F., Hernquist, L., Cox, T.J., Di Matteo, T., Robertson, B., Springel, V., 2005d, ApJ, 632, 81

Hopkins, P.F., Hernquist, L., Cox, T.J., Di Matteo, T., Robertson, B., Springel, V., 2006a, ApJS, 163, 1

Hopkins, P.F., Hernquist, L., Cox, T.J., Robertson, B., Springel, V., 2006b, ApJS, 163, 50

Hopkins, P.F., Hernquist, L., Cox, T.J., Robertson, B., Di Matteo, T., Springel, V., 2006c, ApJ, 639, 700

Hopkins, P.F., Somerville, R., Hernquist, L., Cox, T.J., Robertson, B., Li, Y., 2006d, ApJ, 652, 864

Hopkins, P.F., Hernquist, L., Cox, T.J, Robertson, B., Krause, E., 2007a, ApJ, in press [astro-ph/0707.4005]

Hopkins, P.F., Hernquist, L., Cox, T.J, Robertson, B., Krause, E., 2007b, ApJ, in press |astro-ph/0701351]

Hopkins, P.F., Hernquist, L., Cox, T.J, Keres, D., 2007c, ApJ, submitted [astro-ph/0706.1243]

Hopkins, P.F., Cox, T.J, Keres, D. Hernquist, L., 2007d, ApJ, submitted [astro-ph/0706.1246]

Hopkins, P.F., et al., 2007e, ApJ, in preparation

Hopkins, P.F., et al., 2007f, ApJ, in preparation

Hopkins, P.F., et al., 2007g, ApJ, in preparation

Hopkins, P.F., Richards, G.T., Hernquist, L., 2007h, ApJ, 654, 731

Hopkins, P.F., Hernquist, L., 2006, ApJS, 166, 1 
Iono, D., Ho, P.T.P., Yun, M., Matsushita, S., Peck, A., Sakamoto, K., 2004, ApJ, 616, L63

Iono, D., Yun, M.S., Ho, P.T.P., 2005, ApJS, 158, 1

Iono, D., Wilson, C.D., Takakuwa, S., Yun, M.S., Petitpas, G.R., Peck, A.B.,

Ho, P.T.P., Matsushita, S., Pihlstrom, Y., Wang, Z., 2007, ApJ, 659, 2831

Kauffmann, G., Haehnelt, M.G., 2000, MNRAS, 311, 576

Kennicutt, R., 1998a, ApJ, 498, 541

Kennicutt, R., 1998b, ARA\&A, 36, 189

Keres, D., Yun, M., Young, J., 2003, ApJ, 582, 659

Kim, D.-C., \& Sanders, D.B., 1998, ApJS, 119, 41

Kim, D.-C., Veilleux, S., Sanders, D.B., 2002, ApJS, 143, 277

Komossa, S., Burwitz, V., Hasinger, G., Predehl, P., Kaastra, J.S., Ikebe, Y., 2003, ApJ, 582, L15

Kormendy, J., Fisher, D.B., Cornell, M.E., Bender, R., 2007, ApJ, submitted

Kulesa, C.A., 2002, PhD Thesis, Molecular Hydrogen and its Ions in Dark Interstellar Clouds and Star Forming Regions, University of Arizona

Kulesa, C.A., Hungerford, A.L., Walker, C.K., Zhang, X., Lane, A.P., 2005, ApJ, 625, 194

Lee, H.H., Bettens, R.P.A., Herbst, E., 1996, A\&AS, 111

Li, Y., Hernquist, L., Robertson, B., Cox, T.J., Hopkins P., Springel, V., Gao, L., Di Matteo, T., Zentner, A., Jenkins, A., Yoshida, N., 2007a, ApJ, in press |astro-ph/0608190]

Li, Y., Hernquist, L., Finkbeiner, D., Springel, V., Jiang, L., Fan, X., 2007b, ApJ arXiv:0706.3706

Lidz, A., McQuinn, M., Zaldarriaga, M., Hernquist, L., Dutta, S., 2007, ApJ, submitted [astro-ph/0703667]

Magorrian, J., Tremaine, S., Richstone, D., Bender, R., Bower, G., Dressler, A., Faber, S.M., Gebhardt, K., Green, R., Grillmair, C., Kormendy, J., Lauer, T., 1998, AJ, 115, 2285

Martin, C.L., 2005, ApJ, 621, 227

Mihos, J.C., Hernquist, L.E., 1994a, ApJ, 437, L47

Mihos, J.C., Hernquist, L.E., 1994b, ApJ, 431, L9

Mihos, J.C., Hernquist, L.E., 1996, ApJ, 464, 641

Misawa, T., Eracleous, M., Charlton, J., Tajitsu, A., 2005, ApJ, 629, 115

Misawa, T., Charlton, J.C., Eracleous, M., Ganguly, R., Tytler, D., Kirkman, D., Suzuki, N., Lubin, D., 2007a, ApJ, in press [astro-ph/0702101]

Misawa, T., Eracleous, M., Charlton, J.C., Kashikawa, N., 2007b, ApJ, 660, 152

Morganti, T., Tadhunter, C.N., Oosterloo, T.A., 2005, A\&A, 444, L9

Narayanan, D., Hamann, F., Barlow, T., Burbidge, E.M., Cohen, R.D., Junkkarinen, V., Lyons, R., 2004, ApJ, 601, 715

Narayanan, D., Cox, T.J., Robertson, B., Davé, R., Di Matteo, T., Hernquist, L., Hopkins, P., Kulesa, C., Walker, C.K., 2006a, ApJ, in press astro-ph/0604074

Narayanan, D, Kulesa, C, Boss, A., Walker, C.K., 2006b, ApJ, 647, 1426

Narayanan, D., Li, Y., Cox, T.J., Hernquist, L., Hopkins, P., Chakrabarti, S., Davé, R., Di Matteo, T., Gao, L., Kulesa, C., Robertson, B., Walker, C.K. 2007a, ApJ, in press arXiv:0707.3141

Narayanan, D., Cox, T.J., Shirley, Y., Davé, R., Hernquist, L., Walker, C.K., 2007b, ApJ, in press arXiv:0711.1361

Oppenheimer, B.D., Davé, R., 2006, MNRAS, 373, 1265

Pelupessy, F.I., Papadopoulos, P.P., van der Werf, P., 2006, ApJ, 645, 1024

Pettini, M., Rix, S.A., Steidel, C.C., Hunt, M.P., Shapley, A., Adelberger, K.L., 2002, Ap\&SS, 281, 461

Quinn, P.J., 1984, ApJ, 279, 596

Richards, G.T., et al., 2006, AJ, 131, 2766

Robertson, B., Hernquist, L., Cox, T.J., Di Matteo, T., Hopkins, P.F., Martini, P., Springel, V., 2006a, ApJ, 641, 90

Robertson, B., Bullock, J., Cox, T.J., Di Matteo, T., Hernquist, L., Springel, V., Yoshida, N., 2006b, ApJ, 645, 986

Robertson, B., Cox, T.J., Hernquist, L., Franx, Marijn, Hopkins, P.F., Martini, P., Springel, V., 2006c, ApJ, 641, 21

Robertson, B., Kravtsov, A., 2007, ApJ, in press [arXiv:0710:2102]

Rosolowsky, E., 2005, PASP, 117, 1403

Rosolowsky, E., 2007, ApJ, 654, 240

Rothberg, B., Joseph, R.D., 2004, AJ, 128, 2098

Rothberg, B., Joseph, R.D., 2006, AJ, 131, 185

Rupke, D., Veilleux, S., Sanders, D.B., 2005a, ApJS, 160, 87
Rupke, D., Veilleux, S., Sanders, D.B., 2005b, ApJS, 160, 115

Rupke, D., Veilleux, S., Sanders, D.B., 2005c, ApJ, 632, 751

Rupke, D., Veilleux, S., 2005, ApJ, 631, L37

Sakamoto, K., Scoville, N.Z., Yun, M., Crosas, M,, Genzel, R., \& Tacconi, L.J., 1999, ApJ, 514, 68

Sakamoto, K., Matsushita, S., Peck, A.B., Wiedner, M.C., Iono, D., 2004, ApJ, 616, L59

Sakamoto, K., Ho, P.T.P., Peck, A., 2006, ApJ, 644, 862

Sanders, D.B., Soifer, B.T., Elias, J.H., Neugebauer, G., Matthews, K., 1988a, ApJ, 328, L35

Sanders, D.B., Soifer, B.T., Elias, J.H., Madore, B.F., Matthews, K. Neugebauer, G., Scoville, N.Z., 1988b, ApJ, 325, 74

Sanders, D.B., Scoville, N.Z., Soifer, B.T., 1991, ApJ, 370, 158

Sanders, D.B., Mirabel, I.F., 1996, ARA\&A, 34, 749

Schmidt, M., 1959, ApJ, 129, 243

Schoier, F.L., van der Tak, F.F.S., van Dishoeck, E.F., Black, J.H., 2005, A\&A, 432, 369

Scoville, N., Young, J.S., 1983, ApJ, 265, 148

Scoville, N.Z., Sanders, D.B., Sargent, A.I., Soifer, B.T., Scott, S.L., Lo, K.Y., 1986, ApJ, 311, L47

Scoville, N.Z., Yun, M.S., Bryant, P.M., 1997, ApJ, 484, 702

Scoville, N.Z., Evans, A.S., Thompson, R., Rieke, M., Hines, D.C., Low, F.J., Dinshaw, N., Surace, J.A., Armus, L., 2000, AJ, 119, 991

Shapley, A.E., Steidel, C.C., Pettini, M., Adelberger, K.L., 2003,ApJ, 588, 65

Silk, J., Rees, M.J., 1998, A\&A, 331, L1

Silk, J., Spaans, M., 1997, ApJ, 488, L79

Soifer, B.T., Sanders, D.B., Madore, B.F., Neugebauer, G., Danielson, G.E.,

Elias, J.H., Lonsdale, C.J., Rice, W.L., 1987, ApJ, 320, 238

Solomon, P.M., Rivolo, A.R., Barrett, J., Yahil, A., 1987, ApJ, 319, 730

Solomon, P.M., Downes, D., Radford, S.J.E., 1992, ApJ, 387, L55

Solomon, P.M., Vanden Bout, P., 2005, ARA\&A, 43, 677

Springel, V., 2005, MNRAS, 364, 1105

Springel, V., Hernquist, L., 2002, MNRAS, 333, 649

Springel, V., Hernquist, L., 2003, MNRAS, 339, 312

Springel, V., Hernquist, L., 2005, ApJ, 622, L9

Springel, V., Di Matteo, T., Hernquist, L., 2005a, MNRAS, 361, 776

Springel, V., Di Matteo, T., Hernquist, L., 2005b, ApJ, 620L, 79

Tacconi, L.J., Neri, R., Chapman, S.C., Genzel, R., Smail, I., Ivison, R.J., Bertoldi, F., Blain, A., Cox, P., Greve, T., Omont, A., 2006, ApJ, 640, 228

Tran, Q.D., Lutz, D., Genzel, R., Rigopoulou, D., Spoon, H.W.W., Sturm, E., Gerin, M., Hines, D.C., Moorwood, A.F.M., Sanders, D.B., Scoville, N., Taniguchi, Y., Ward, M., 2001, ApJ, 552, 527

Tremonti, C.,A., Moustakas, J., Diamond-Stanic, A.M., 2007, ApJ, in press [arXiv0706.0527]

Trump, J.R., Hall, P..B., Reichard, T.A., Richards, G.T., Schneider, D.P., Vanden Berk, D.E., Knapp, G.R., Anderson, S.F., Fan, X., Brinkman, J., Kleinman, S.J., Nitta, A., 2006, ApJS, 165, 1

van Zadelhoff, G-J., Dullemond, C., van der Tak, F.F.S., Yates, J.A., Doty, S.D., Ossenkopf, V., Hogerheijde, M.R., Juvela, M., Wiesemeyer, H., Schoier, F.L, 2002, A\&A, 395, 373

Veilleux, S., Kim, D.-C., Sanders, D.B., 2002, ApJS, 143, 315

Veilleux, S., Cecil, G., Bland-Hawthorn, J., 2005, ARA\&A, 43, 769

Wada, K., Tomisaka, K., 2005, ApJ, 619, 93

Walker, C.K., Adams, F.C., Lada, C.J., 1990, ApJ, 349, 515

Walter, F., Weiß, A., Scoville, N., 2002, ApJ, L21-L25

Ward-Thompson, D., Scott, P.F., Hills, R.E., Andre, P., 1994, MNRAS, 268, 276

Weil, M., Hernquist, L., 1992, Nature, 358, 734

Weil, M., Hernquist, L., 1993, ApJ, 405, 142

Weiß, A., Downes, D., Walter, F., Henkel, C., 2005a, A\&A, 440,L45

Weiß, A., Walter, F., Scoville, N.Z., 2005b, A\&A, 438, 533

Weiß, A., Downes, D., Neri, R., Walter, F., Henkel, C., Wilner, D.J., Wagg, J., Wiklind, T., 2007, A\&A, in press [arXiv:astro-ph/0702669]

Wise, J.H., Eracleous, M., Charlton, J., Ganguly, R., 2004, ApJ, 613, 129

Yao, L., Seaquist, E.R., Kuno, N., Dunne, L., 2003, ApJ, 588, 771 